\title{
Automatic segmentation of abnormal capillary nonperfusion regions in optical coherence tomography angiography images using marker- controlled watershed algorithm
}

Razieh Ganjee

Mohsen Ebrahimi Moghaddam

Ramin Nourinia 


\title{
Automatic segmentation of abnormal capillary nonperfusion regions in optical coherence tomography angiography images using marker-controlled watershed algorithm
}

\author{
Razieh Ganjee, ${ }^{a}$ Mohsen Ebrahimi Moghaddam, ${ }^{a, \star}$ and Ramin Nourinia ${ }^{b}$ \\ ashahid Beheshti University G.C, Faculty of Computer Science and Engineering, Tehran, Iran \\ ${ }^{b}$ Shahid Beheshti University of Medical Sciences, Ophthalmic Research Center, Tehran, Iran
}

\begin{abstract}
Diabetic retinopathy (DR) is one of the most complications of diabetes. It is a progressive disease leading to significant vision loss in the patients. Abnormal capillary nonperfusion (CNP) regions are one of the important characteristics of DR increasing with its progression. Therefore, automatic segmentation and quantification of abnormal CNP regions can be helpful to monitor the patient's treatment process. We propose an automatic method for segmentation of abnormal CNP regions on the superficial and deep capillary plexuses of optical coherence tomography angiography (OCTA) images using the marker-controlled watershed algorithm. The proposed method has three main steps. In the first step, original images are enhanced using the vesselness filter and then foreground and background marker images are computed. In the second step, abnormal CNP region candidates are segmented using the marker-controlled watershed algorithm, and in the third step, the candidates are modeled using an undirected weighted graph and finally, by applying merging and removing procedures correct abnormal CNP regions are identified. The proposed method was evaluated on a dataset with 36 normal and diabetic subjects using the ground truth obtained by two observers. The results show the proposed method outperformed some of the state-of-the-art methods on the superficial and deep capillary plexuses according to the most important metrics. @ 2018 Society of Photo-Optical Instrumentation Engineers (SPIE) [DOI: 10.1117/1 JBO.23.9.096006]
\end{abstract}

Keywords: diabetic retinopathy; capillary nonperfusion; optical coherence tomography angiography; marker-controlled watershed algorithm.

Paper 180251RR received Apr. 30, 2018; accepted for publication Sep. 5, 2018; published online Sep. 27, 2018.

\section{Introduction}

Diabetic retinopathy (DR) is a progressive disease and one of the leading causes of blindness in the world. However, significant cases of vision loss can be prevented by the diagnosis of DR in early stages. ${ }^{1-3}$ Based on the early treatment of DR study, DR is classified into four stages, including mild nonproliferative diabetic retinopathy (NPDR), moderate NPDR, severe NPDR, and proliferative diabetic retinopathy (PDR). ${ }^{4}$ Depending on the progression of the DR, various vascular abnormalities are characterized. ${ }^{5,6}$ Capillary nonperfusion $(\mathrm{CNP})$ regions or macular ischemia is one of the DR key features found in early stages and increases with the severity of DR. ${ }^{7,8}$ Therefore, automatic detection and quantification of macular ischemia as a valuable biomarker for prediction and monitoring DR progression can be helpful. ${ }^{9-11}$

Color fundus photography and fluorescein angiography (FA) are widely used in retinal vessels analysis and lesion detection in macular diseases. ${ }^{12-14} \mathrm{FA}$ is a standard tool to evaluate retinal vascular changes. Although this imaging method is still used for grading DR, it is time consuming, invasive, and has some side effects on patients, including vomiting, skin and eye discoloration, urticaria, and, in rare cases, severe anaphylactic reactions. ${ }^{15}$ Optical coherence tomography (OCT) is an important noninvasive tool commonly used to visualize structural

*Address all correspondence to: Mohsen Ebrahimi Moghaddam, E-mail: m_moghadam@sbu.ac.ir changes of the retina caused by macular diseases, such as cystoid edema, intraretinal and subretinal fluid. ${ }^{16-18}$ It allows three-dimensional (3-D) cross-sectional imaging of the retina at the micron-scale resolution. ${ }^{16}$ Due to the poor vasculature contrast in conventional OCT images, properly assessing vascular changes in these images is not possible. Optical coherence tomography angiography (OCTA) as an extension of the OCT imaging modality provides 3-D imaging of the retinal microvasculature. ${ }^{15,19}$ In comparison with the conventional angiography imaging modality (FA), OCTA is fast, noninvasive without any side effects, and can be repeated multiple times on the same day. ${ }^{20,21}$ It also allows visualizing vascular abnormality at specific depths, including superficial, deep, outer retina, and choriocapillaris plexuses, which is not possible in FA as a two-dimensional (2-D) modality. The ability of OCTA imaging in the visualization of vascular abnormality in different layers makes it a promising tool in detection and monitoring vascular changes in retinal disease such as DR. ${ }^{15,22-26}$ Macular ischemia or CNP regions as an early valuable biomarker is clear in OCTA superficial and deep plexus. ${ }^{9-11}$

There are a few attempts to automatic quantification of CNP regions in literature. Hwang et al. ${ }^{11}$ proposed a threshold-based approach to segment CNP regions. They investigated the total area of macula ischemia in DR cases compared with a normal group and showed that this area is significantly higher in DR cases. This method was improved by Zhang et al. ${ }^{9}$ In their

$1083-3668 / 2018 / \$ 25.00$ @ 2018 SPIE 


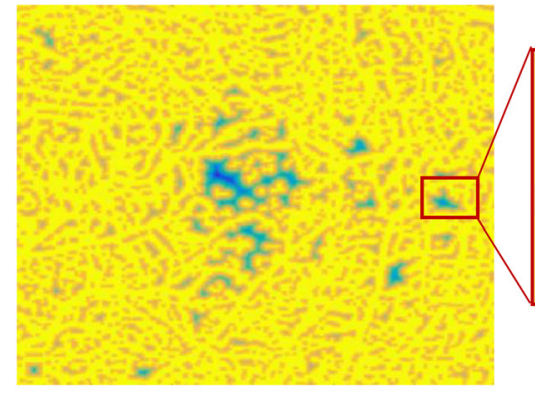

(a)

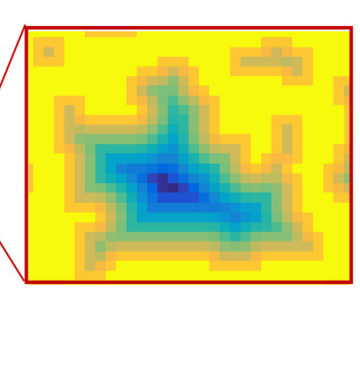

(b)

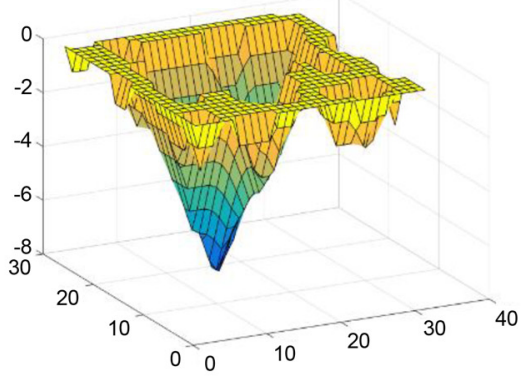

(c)

Fig. 1 The mesh plot of an abnormal CNP region in negative VD image. (a) The VD image with negative values, (b) one of the abnormal CNP region in (a), and (c) the mesh plot of (b).

method, first, a projection resolved algorithm ${ }^{27}$ was used to segment the OCTA images into three vascular plexuses, namely superficial, intermediate, and deep. Then, segmentation of CNP regions was done in three steps consisting of preprocessing, vessel distance (VD) transform, and morphological operations. In the preprocessing step, the contrast of the input image was enhanced using vesselness filter. ${ }^{28}$ In the second step, using local thresholding, the vessel network was extracted and then VD map was obtained by applying Euclidean distance transform to the binary vessel network. Finally, by thresholding the VD map and applying morphological operations, abnormal CNP regions were extracted. They quantified the segmented regions by computing the total area of them with and without the foveal avascular zone (FAZ) area and showed that this total area can be a valuable measure to detect mild NPDR group from the normal group. These threshold-based methods cannot give an accurate abnormal CNP regions map because the boundary of the regions is limited to the range allowed by the threshold value.

A baseline and simple approach for the automatic segmentation of CNP regions was used in Schottenhamml et al. ${ }^{10}$ study. In this approach, first, the vessel map is obtained, and then each nonvessel pixel in the logical vessel map is considered as a CNP region. They used the same approach as Zhang et al. ${ }^{9}$ to segment vessel network and extracted the vessel network by local thresholding of the enhanced input image. Then, the CNP regions were obtained by finding the connected components of the inverted binary image. In this method, the means of 10 and 20 segmented regions with and without the FAZ were computed. They showed that the mean of 10 largest regions can be a useful measure for determining disease status in patients with diabetes mellitus and DR. It should be mentioned that Schottenhamml et al. ${ }^{10}$ focused on the extraction of new parameters for nonperfusion quantification rather than the segmentation of the whole abnormal CNP regions. However, roughly separating the 10 largest regions as abnormal CNPs could lead to loss of some abnormalities for a patient group and an increase in false positives for normal subjects.

In the present paper, we tried to localize and segment abnormal regions accurately and finally provide an accurate map of them for further medical analyses and quantifications. Normally, there is a regular distance between retinal vessel pixels. With the loss of blood vessels due to the ocular diseases, this distance increases and creates abnormal holes between the vessel structures. Therefore, in the medical field, abnormal CNP regions are defined as holes with abnormal retinal VD. The previous works ${ }^{9-11}$ have not been operated close to this concept of abnormal CNP regions and have been focused more on the accurate vessel network segmentation. In these works, by segmenting vessel network as foreground objects, the input image is divided into background and foreground and then the background is considered to segment abnormal CNP regions. On the other hand, the previous methods ${ }^{9-11}$ mostly tended to investigate the impact of the ocular disease on changing CNP regions. In these methods, accurate positioning of the CNP regions has not been considered and only computing the total area of them is important. Therefore, the method that they used for segmentation of abnormal regions cannot provide an accurate map of abnormal CNP region positions.

Since the functionality of the watershed algorithm is close to the concept of CNP regions, we proposed a marker-controlled watershed algorithm in a proper framework for the segmentation of abnormalities. This fact was shown in Fig. 1. In the topographic representation of the grayscale image, where high intensity shows peaks and hills and low intensity shows valleys, the watershed algorithm finds the lines that surrounded each catchment basins or isolated valleys (local minima). As shown in Fig. 1(c), in the mesh plot of one of the abnormal CNP region in the retinal VD image with negative values, CNP regions are appeared as catchment basins or isolated valleys that can be extracted using the watershed algorithm.

Considering the postprocessing step that consists of merging and removing procedures, it shows that the proposed method is more flexible and extensible compared to the previous methods ${ }^{9-11}$ because it is possible to extend and improve the proposed method by changing the similarity and removing measures based on the new finding in the medical investigation of OCTA images. This aspect will be explained more in the discussion section.

The results show that the proposed method can segment abnormal CNP regions up to their boundary more accurately than some other methods.

The rest of the paper has been organized as follows: the proposed method is presented in Sec. 2. The evaluation of the proposed method and discussion are discussed in Secs. 3 and 4, respectively. And finally, the paper is concluded in Sec. 5.

\section{Proposed Method}

The watershed algorithm is widely used in the medical image segmentation, such as lesion segmentation of breast in mammogram ${ }^{29}$ and ultrasound images, ${ }^{30,31}$ intraretinal cysts segmentation in OCT images, ${ }^{32}$ lymphoma segmentation in computerized tomography (CT) images, ${ }^{33}$ and malignant lesion segmentation in images acquired by magnetic resonance imaging. ${ }^{34}$ 

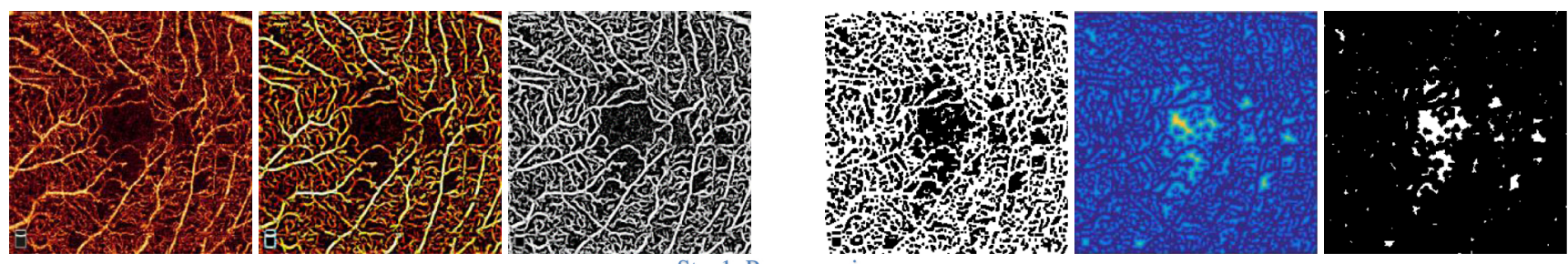

Step1: Preprocessing

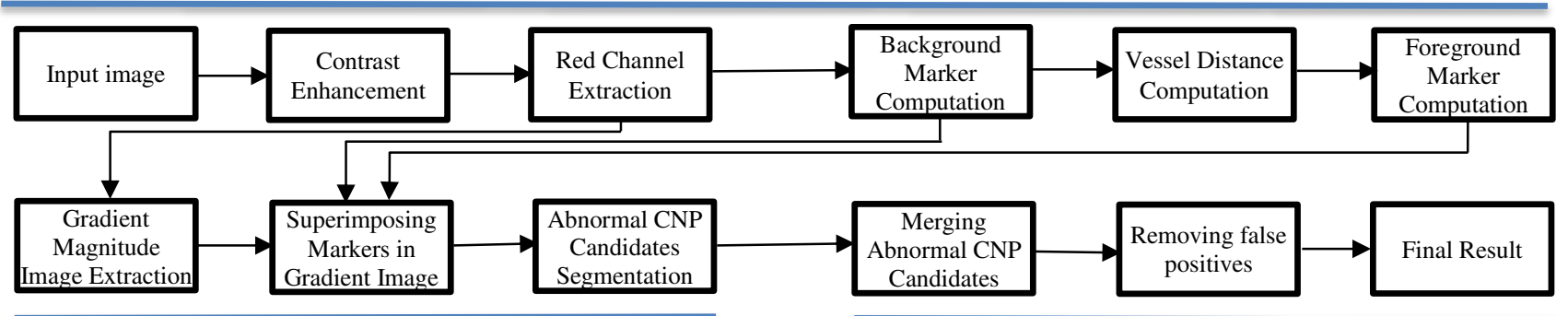

Step2: Marker-Controlled Watershed Segmentation
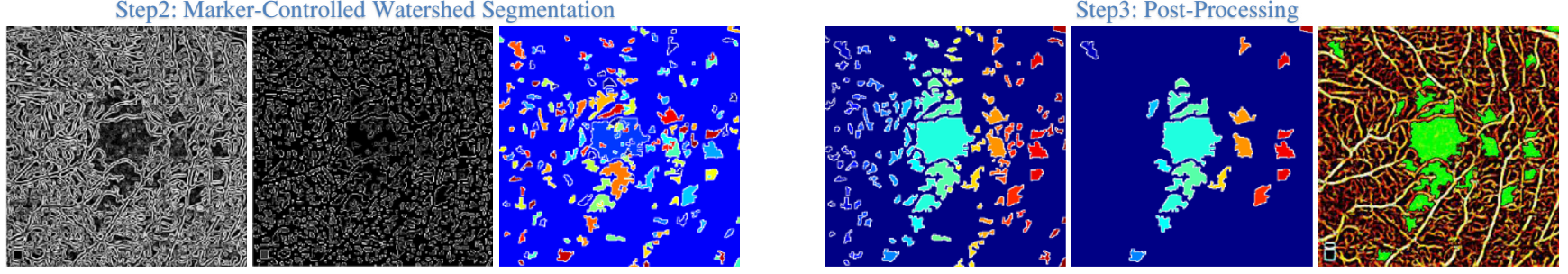

Fig. 2 The block diagram of the proposed method.

The watershed algorithm can be an effective segmentation method if it is used with auxiliary processing before and after segmentation. Oversegmentation is the main drawback of the conventional watershed algorithm. ${ }^{35-37}$ In this study, we managed this problem using preprocessing and postprocessing steps before and after the segmentation.

As shown in Fig. 2, the proposed method has three main steps: first, preprocessing to enhance the input image and compute marker images; second, marker-controlled watershed segmentation to segment the abnormal CNP region candidates; and third, postprocessing to identify the correct abnormal CNP regions. In the following, each step is described in detail.

\subsection{Preprocessing}

The preprocessing step contains two main processes, namely, first, increasing the contrast of the input image and second, computing the foreground and background marker images.

\subsubsection{Increasing the contrast of the input image}

Noisy images have negative effects on the watershed algorithm and cause oversegmentation. In this study, we used the Optovue OCTA device for imaging. Using this device, enface images can be extracted in color and grayscale modalities. In this step, first, the red channel of the input image was extracted because, as is shown in Fig. 3, this channel has the highest contrast between the vessel pixels and background in comparison with the grayscale image, red and blue channels provided by the Optovue system. Then, we applied two-scale ( $\sigma=1$ and $\sigma=2$ pixels) vessel enhancement filtering to remove background noise from the red channel of the input image and enhance the contrast between the vessel and background pixels. ${ }^{28}$ The vesselness measure $V_{0}$ obtained by applying the vesselness filter on the red channel of a superficial image is shown in Fig. 4(b). As it is obvious, the contrast between the flow pixels and background has been increased.

\subsubsection{Computing the foreground and background marker images}

In the marker-controlled watershed algorithm, two logical marker images, including foreground and background markers, should be specified. The foreground marker images should contain connected components of nonzero pixels within each of the desired objects (abnormal CNP regions). Also, the background marker should contain nonzero pixels that are not part of any desired objects (vessel pixels). Therefore, the foreground marker image $f_{f g m}(x, y)$ is defined as a binary image with the connected components of pixels inside the CNP regions set to 1 and the remained pixels set to 0 . Also, the background marker image $f_{\text {bgm }}(x, y)$ is a binary image with the pixels that are not part of any CNP regions (vessel pixels) set to 1 .

By applying Otsu ${ }^{38}$ threshold value $T_{1}$ followed by closing morphological operation with structuring element $B(3 \times 3$ pixel square) on $V_{0}$; the background marker image $f_{b g m}(x, y)$ is computed. That is,

$f_{\text {bgm }}(x, y)=\varepsilon_{B}\left[\delta_{B}\left(V_{0}^{T_{1}}\right)\right]$,

where $\varepsilon$ and $\delta$ are morphological erosion and dilation operator, respectively (closing is a dilation followed by an erosion). $V_{0}^{T_{1}}$ is the result of applying Otsu threshold value $T_{1}$ on $V_{0}$. The background marker image is shown in Fig. 4(c). The shape and size of the structuring element were chosen empirically. Using a larger element eliminates the useful information from the image, and the small holes may not be extracted. 


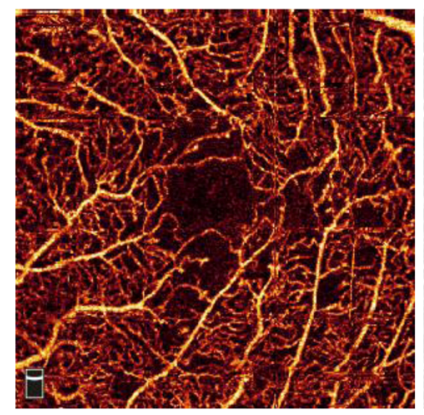

(a)

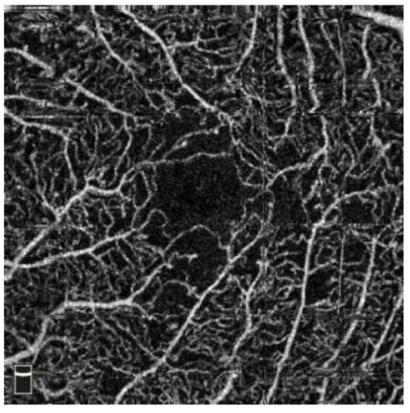

(b)

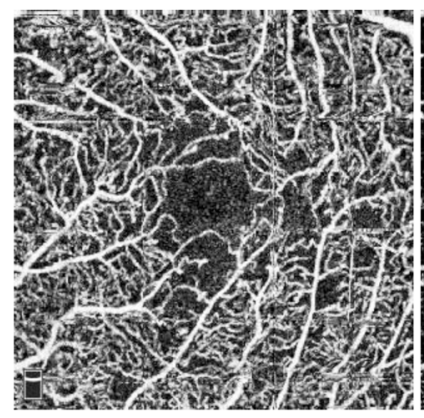

(c)

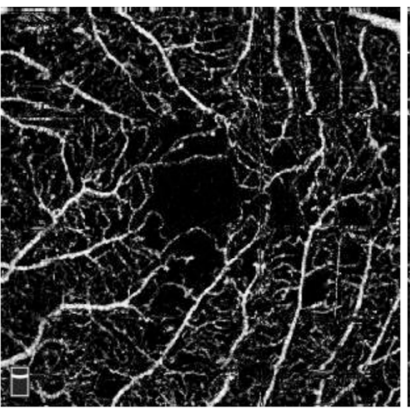

(d)

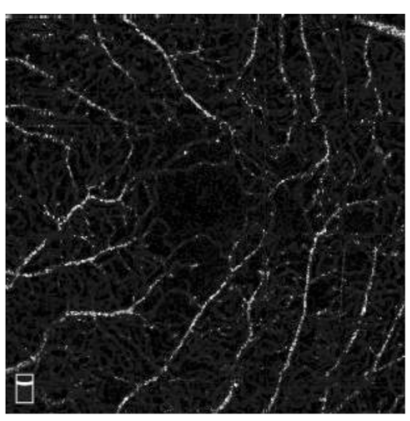

(e)

Fig. 3 A visual comparison among the contrast of different variants of the input image (the provided grayscale image and three RGB channels). (a) RGB image, (b) the provided grayscale image, (c) red channel, (d) green channel, and (e) blue channel.

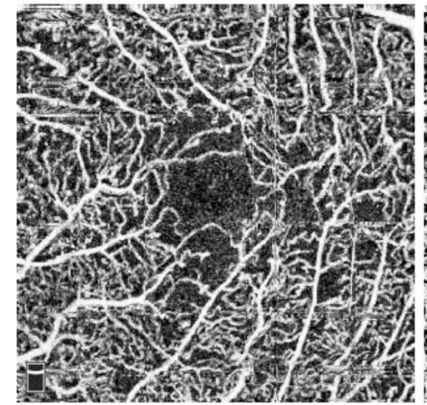

(a)

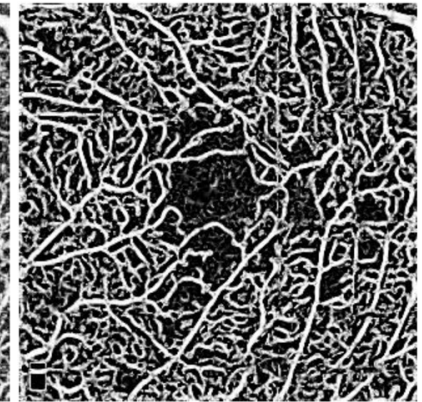

(b)

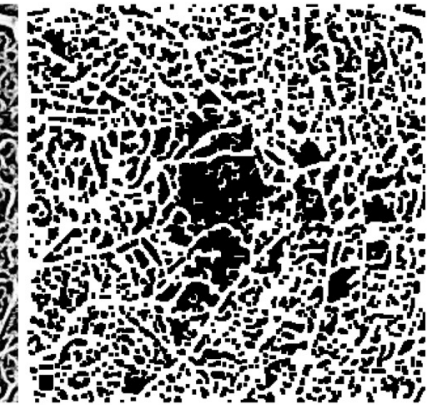

(c)

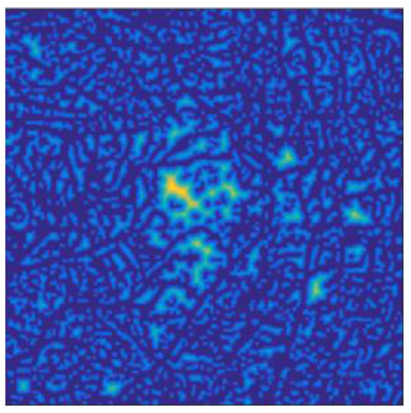

(d)

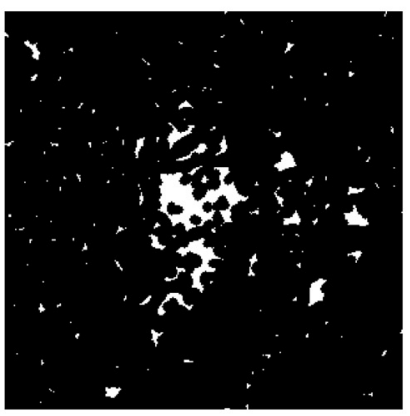

(e)

Fig. 4 Preprocessing step. (a) Red channel in Fig. 3(c), (b) Enhanced image (vesselness measure), (c) background marker image, (d) distance map image, and (e) foreground marker image.

Then, the Euclidean distance function was used to compute the foreground marker image because abnormal CNP regions are defined as holes with abnormal VD. Therefore, to provide the foreground marker image, first, the VD map $f_{v d m}(x, y)$ is obtained by computing the distance of each pixel from the nearest nonzero pixel of background marker image ${ }^{39}$ as follows:

$$
f_{v d m}(x, y)=\min _{\left(x^{\prime}, y^{\prime}\right)=\left\{(x, y) \mid f_{b g m}(x, y)=1\right\}} \operatorname{ED}\left[(x, y),\left(x^{\prime}, y^{\prime}\right)\right],
$$

where ED is Euclidean distance function. Figure 4(d) shows the VD map image computed by Eq. (2). Then, by applying 


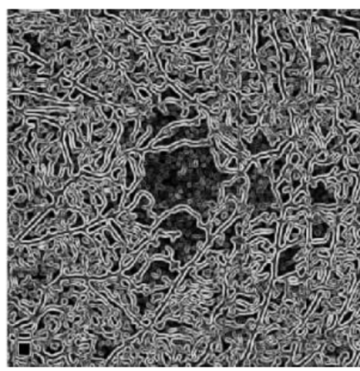

(a)

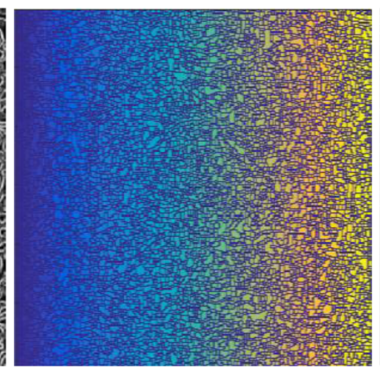

(b)

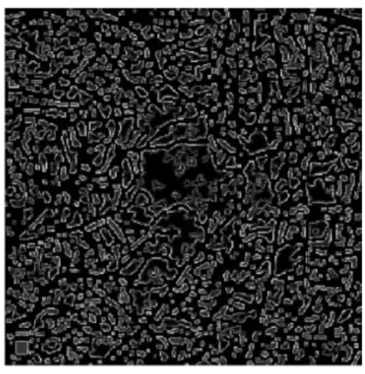

(c)

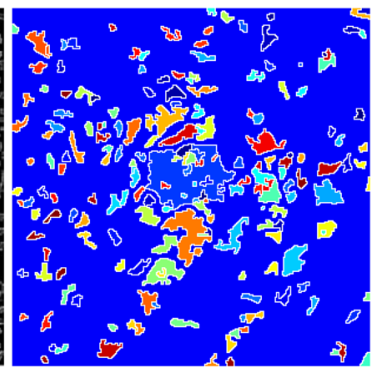

(d)

Fig. 5 The output of applying the marker-controlled watershed to the gradient magnitude of Fig. 3(b).

(a) The gradient magnitude of Fig. 3(b), (b) applying the conventional watershed algorithm to (a),

(c) imposing marker image [Figs. 4(c) and 4(e)] in (a), and (c) the marker-controlled watershed result.

threshold value $T_{2}$ to the VD image, the foreground marker image is computed as follows:

$f_{f g m}(\mathrm{x}, \mathrm{y})=\left\{\begin{array}{ll}1, & f_{v d m}(x, y) \geq T_{2} \\ 0, & f_{v d m}(x, y)<T_{2}\end{array}\right.$.

Figure 4(e) shows the result of applying threshold value $T_{2}$ on $f_{v d m}$. The equation used for computing this threshold value will be explained in Sec. 2.4. According to this equation [Eq. (13)], the threshold value $T_{2}$ is equal to 3 pixels. If the threshold $T_{2}$ is considered higher than 3 pixels, some abnormal CNPs may not be nominated in the foreground image or may not be delineated up to their true boundary in the final step. And if the threshold value $T_{2}$ is considered lower than 3 pixels, normal regions may be nominated along with abnormalities.

\subsection{Marker-Controlled Watershed Segmentation}

The watershed algorithm by immersion was used to segment abnormal CNP region candidates. In the following, we first present a brief description of the watershed algorithm by immersion and then we describe how it has been adjusted in the form of the marker-controlled watershed to segment CNP regions up to their true boundaries.

\subsubsection{Watershed algorithm by immersion}

In this algorithm, the input image is considered as a topographic surface and the regional minimum points are found. Supposing both minima want to be merged, a dam is created to avoid merging. By continuing this immersion process, we finally reach a stage, where only the upper parts of the dams can be seen on the surface. The boundaries created by the corresponding dams are the dividing lines of the watersheds. Therefore, these are continuous boundaries extracted by the watershed algorithm.

\subsubsection{Applying watershed algorithm to segment CNP region candidates}

Generally, watershed algorithm is applied to the gradient image $^{37}$ because in the gradient image, objects appear as dark regions and their boundaries are located at pixels with high gradient values. The Sobel operator is one of the well-known and simple filters in terms of computation for obtaining gradient magnitude of the image. Using this filter, the gradient magnitude of the image $f(x, y)$ is computed as follows: ${ }^{40}$

$$
f_{g}(x, y)=\sqrt{\left[G_{x} * f(x, y)\right]^{2}+\left[G_{y} * f(x, y)\right]^{2}},
$$

where $G_{x}$ and $G_{y}$ are Sobel edge masks in $X$ and $Y$ directions, respectively. Figure 5(a) shows the gradient magnitude of Fig. 4(b).

Due to the existence of numerous regional minima in the gradient image, directly applying watershed algorithm to the gradient image caused oversegmentation. Figure 5(b) shows the result of applying the watershed algorithm to the gradient magnitude image in Fig. 5(a). To overcome this intrinsic drawback of the watershed algorithm, the marker-controlled watershed algorithm was used. Using this technique, the number of regional minima was controlled by defining marker image and thus flooding only occurred in basins corresponding to each marker. ${ }^{41}$ Therefore, in the marker-controlled watershed, first, the marker points should be computed and then these markers should be superimposed on the gradient image as global minimums.

Based on the foreground marker image $f_{f g m}(x, y)$ and the background marker image $f_{b g m}(x, y)$ provided by the preprocessing step, the marker image $f_{m}(x, y)$ is defined as follows:

$f_{m}(x, y)=f_{f g m}(x, y) \cup f_{b g m}(x, y)$.

Then, minima imposition morphological operation was used to superimpose each marker on the gradient image. ${ }^{40}$ In this operation, as it is defined by Eq. (6), first, the pointwise minimum between the gradient image $f_{g}$ and the marker image $f_{m}$ is computed $\left(f_{g} \wedge f_{m}\right)$, then using morphological reconstruction by erosion, the minimum superimposed gradient image $f_{m s g}(x, y)$ is obtained [Fig. 5(c)]:

$f_{m s g}(x, y)=R_{f_{g} \wedge f_{m}}^{\varepsilon}\left[f_{m}(x, y)\right]$,

where $R^{\varepsilon}$ is the morphological reconstruction by erosion of $f_{g} \wedge f_{m}$ from the marker image $f_{m}$. In fact, the pointwise minimum forces each region in the gradient image that is corresponded with each white region in the marker image to be a global minimum of the gradient image. Then, all gradient image information except the masked regions (global minimums) are recovered using the morphological reconstruction by erosion.

Finally, CNP region candidates were obtained by applying the watershed algorithm to the superimposed gradient image $f_{m s g}(x, y)$. Figure $5(\mathrm{~d})$ shows the result of applying the watershed algorithm to $f_{m s g}$. 


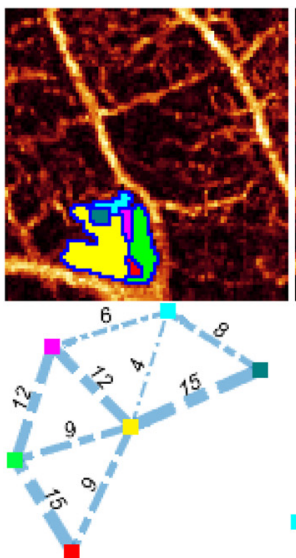

(a)
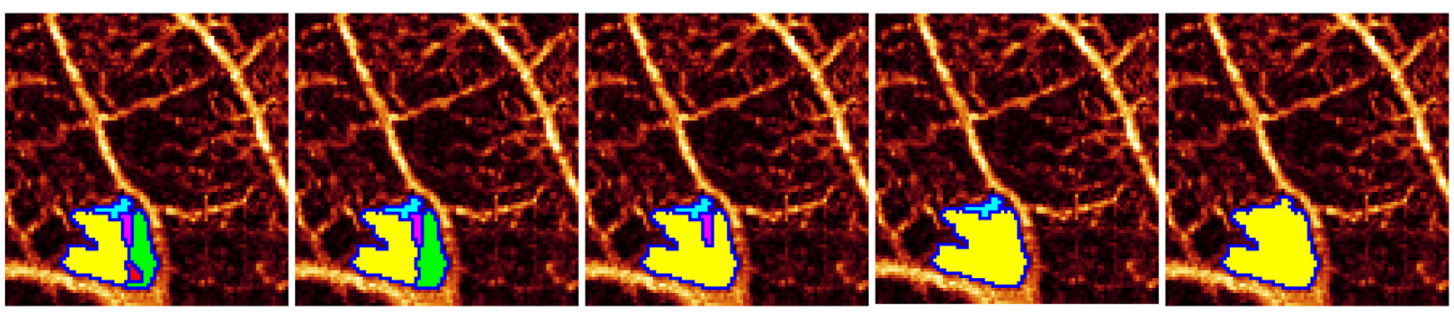

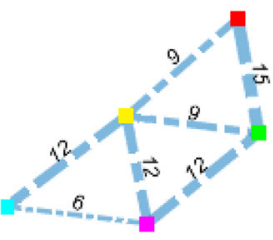

(b)

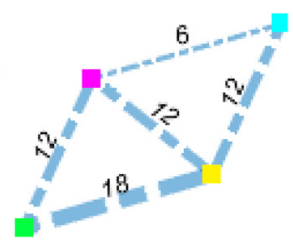

(c)

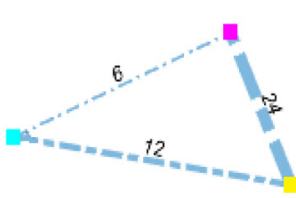

(d)

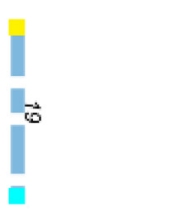

(e)

Fig. 6 (a-f) Representation of the graph-based merging process on a sample of fragmented abnormal CNP candidates.

\subsection{Postprocessing}

As shown in Fig. 5(d), some of the segmented CNP regions are fragmented and do not show an accurate range of abnormal region. In addition, some of the normal regions were segmented along with abnormalities. Therefore, the postprocessing step was considered to modify the marker-controlled watershed segmentation output and obtain correct abnormal CNP region with more accurate boundary. The postprocessing step was performed by merging fragmented candidates based on the proper similarity measure and then separating correct abnormal CNP regions from modified candidates.

To merge the fragmented regions, the abnormal CNP region candidates were modeled as an undirected weighted graph $G=(V, E, w)$ so that by defining $V=\left\{R_{i}\right\}_{i=1}^{n}$, each vertex represents a candidate region $R_{i}$ and each edge $e_{i}=\left(R_{i}, R_{j}\right)$ in $E=\left\{e_{i}\right\}_{i=1}^{m}$ connects two neighboring candidates. $n$ and $m$ are equal to the number of candidates and the number of neighbors, respectively. By considering the weight of each edge equal to the size of the common boundary between two neighboring regions, the weighting function $w: E \rightarrow \mathbb{N}$ for each two neighboring vertexes $R_{i}$ and $R_{j}$ is defined as follows:

$w\left(R_{i}, R_{j}\right)=\left|\left[R_{i}-\varepsilon_{B}\left(R_{i}\right)\right] \cap\left[R_{j}-\varepsilon_{B}\left(R_{j}\right)\right]\right|$,

where $\varepsilon$ is the morphological erosion and $B(3 \times 3$ pixel square) is the structuring element. The shape and size of the structuring element were chosen empirically. Using a larger element leads to a wider border extraction that is not suitable for our purpose. The notation || shows the number of pixels in the common

boundary computed by $\left[R_{i}-\varepsilon_{B}\left(R_{i}\right)\right] \cap\left[R_{j}-\varepsilon_{B}\left(R_{j}\right)\right]$. Due to the discontinuity of CNP candidates, the computed weighted graph consists of a set of subgraphs $G_{i}^{\prime}$ as follows:

$$
\begin{aligned}
G & =(V, E, w) \\
& =\bigcup_{i=1}^{l}\left\{G_{i}^{\prime}\left(V_{i}^{\prime}, E_{i}^{\prime}, w_{i}^{\prime}\right) \mid V_{i}^{\prime} \subseteq V, E_{i}^{\prime} \subseteq E, w_{i}^{\prime} \subseteq w\right\},
\end{aligned}
$$

where $l$ is the number of discontinuities.

Therefore, the merging process was accomplished for each subgraph $G_{i}^{\prime}$ independently. Two neighboring regions $R_{i}$ and $R_{j}$ are similar if their common boundary is larger than threshold value $T_{3}$. According to Sec. 2.4 , this threshold value was computed equal to 6 pixels. Thus, the largest edge in each subgraph is extracted and the threshold condition is checked. If the condition is satisfied, the nodes associated with this edge merge together. It means that the same label is given to the corresponding regions of those two nodes and the boundary between them is removed. Then, the subgraphs are updated and rebuilt. Considering $R_{i}$ as a region, it should be removed and merged with $R_{j}$, the components $V^{\prime}, E^{\prime}$, and $w^{\prime}$ of each subgraph $G^{\prime}$ are updated as follows:

$$
\begin{aligned}
V_{t+1}^{\prime}= & V_{t}^{\prime}-R_{i}, \\
E_{t+1}^{\prime}= & E_{t}^{\prime}-\left\{\left(R_{i}, b\right) \mid b \in V_{t}^{\prime}-R_{i},\left(R_{i}, b\right) \in E_{t}^{\prime}\right\} \\
& +\left\{\left(R_{j}, b\right) \mid b \in V_{t}^{\prime}-\left\{R_{i}, R_{j}\right\},\left(R_{i}, b\right) \in E_{t}^{\prime}\right\},
\end{aligned}
$$

$w_{t+1}^{\prime}\left(R_{j}, b\right)= \begin{cases}w_{t}^{\prime}\left(R_{j}, b\right)+w_{t}^{\prime}\left(R_{i}, b\right) & \text { if } w_{t}^{\prime}\left(R_{j}, b\right) \in E_{t}^{\prime} \text { and } w_{t}^{\prime}\left(R_{i}, b\right) \in E_{t}^{\prime} \\ w_{t}^{\prime}\left(R_{i}, b\right) & \text { if } w_{t}^{\prime}\left(R_{j}, b\right) \notin E_{t}^{\prime} \text { and } w_{t}^{\prime}\left(R_{i}, b\right) \in E_{t}^{\prime}, \\ w_{t}^{\prime}\left(R_{j}, b\right) & \text { if } w_{t}^{\prime}\left(R_{j}, b\right) \in E_{t}^{\prime} \text { and } w_{t}^{\prime}\left(R_{i}, b\right) \notin E_{t}^{\prime}\end{cases}$

where $V_{t+1}^{\prime}, E_{t+1}^{\prime}$, and $w_{t+1}^{\prime}$ are updated sets of $V_{t}^{\prime}, E_{t}^{\prime}$, and $w_{t}^{\prime}$ in the step $t+1$ of merging procedure.

The merging process termination condition is defined as reaching unified regions or missing thresholding condition. Figures 6(a)-6(f) show the merging process on a sample of fragmented CNP candidates. As it is seen, first two yellow and dark green regions in Fig. 6(a), which have the largest common boundary, are merged in Fig. 6(b). By this merging, the dark green region is included in the yellow region so that the boundary of the yellow region changed and the corresponding weighted graph is updated. By continuing this procedure, the unified yellow region in Fig. 6(f) is obtained. Figure 7(a) 


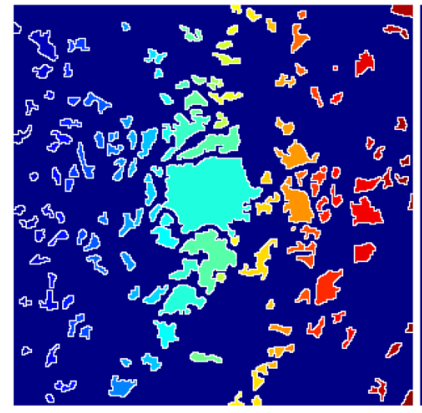

(a)

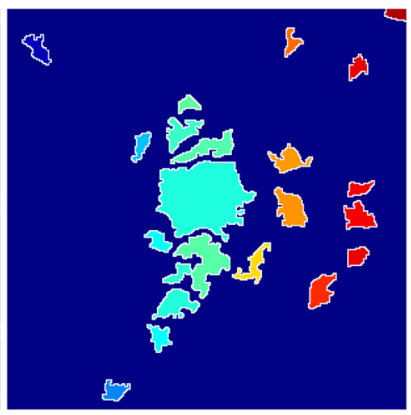

(b)

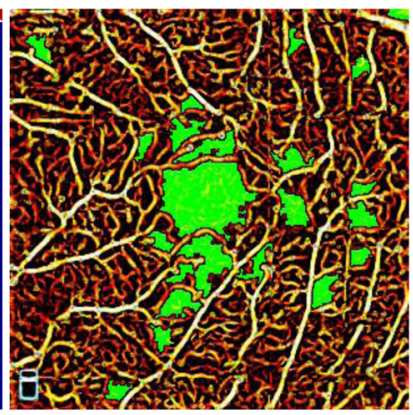

(c)

Fig. 7 Postprocessing step. (a) Merging fragmented abnormal CNP candidates of Fig. 5(d), (b) separating correct abnormal CNP regions from (a), and (c) mapping abnormal CNP regions on the color enhanced input image.

shows the result of applying the graph-based merging algorithm to the marker-controlled watershed segmentation output in Fig. 5(d).

Finally, to remove normal regions that are wrongly extracted along with the abnormalities, a circle-checking step with a circle of radius $R$ is considered. So, correct abnormal CNP regions are obtained by removing any CNP candidates where it was not possible to draw a circle of radius $R$ inside them. A circle of radius $R$ can be inscribed in each abnormal region because of using Euclidean distance function and the way of computing VD (the distance of each pixel from the nearest nonzero pixel). The radius $R$ was considered as 4 pixels. It is computed by Eq. (12) that will be described in Sec. 2.4.

Figures 7(b) and 7(c) show the final abnormal CNP regions and their mapping on the color enhanced input image, respectively. The color enhanced input image was obtained by applying vesselness filter to each red, blue, and green channels independently.

\subsection{Determination of the Threshold Values}

According to the analysis of VD, values in the OCTA images of normal and diabetic subjects, the threshold values $T_{2}$ in the preprocessing step, $T_{3}$ in the merging algorithm, and the radius $R$ of the circle in the final step are computed as follows:

$$
\begin{aligned}
R= & \min \{d \mid d \in\{1, \ldots, \mathrm{MVD}\} \text { and } \\
& \left.\frac{\left(\mathrm{MPFD}_{\mathrm{VD} \geq d}-\mathrm{MPFN}_{\mathrm{VD} \geq d}\right)}{\mathrm{MPFD}_{\mathrm{VD} \geq d}}>\mathrm{CL}_{1}\right\}, \\
T_{2}= & \min \{d \mid d \in\{1, \ldots, \mathrm{MVD}\} \text { and } \\
& \left.\frac{\left(\mathrm{MPFD}_{\mathrm{VD} \geq d}-\mathrm{MPFN}_{\mathrm{VD} \geq d}\right)}{\mathrm{MPFD}_{\mathrm{VD} \geq d}}>\mathrm{CL}_{2}\right\},
\end{aligned}
$$

$T_{3}=2 T_{2}$,

where MPFD and MPFN are the mean of the pixel frequency with specific VD in each OCTA image of normal and diabetic subjects, respectively. MVD is maximum vessel distance. $\mathrm{CL}_{1}$ and $\mathrm{CL}_{2}$ are confidence levels equal to 0.99 and 0.90 , respectively. Table 1 shows the values of MPFD, MPFN, and $\frac{\left(\mathrm{MPFD}_{\mathrm{VD}}-\mathrm{MPFN}_{\mathrm{VD}}\right)}{\mathrm{MPFD}_{\mathrm{VD}}}$ for $\mathrm{VD} \geq d, \mathrm{~d} \in\{1, \ldots, \mathrm{MVD}\}$. As can be seen, using the above equations, the threshold values $T_{2}, T_{3}$, and the radius $R$ are equal to 3,6, and 4 pixels, respectively.

The radius $R$ equal to 4 pixels $(40 \mu \mathrm{m})$ is a discriminator between normal and abnormal CNP regions. Due to the noise and functionality of the watershed algorithm, some of the abnormal CNP regions are not extracted up to their true boundary. Therefore, using threshold value $T_{2}$ less than $R$ $\left(T_{2}=3 \leq R\right)$, the regions with less VD value are extracted. Finally, in the merging step, if these regions have a common border size larger than or equal to $T_{3}=2 T_{2}=6$ pixels with abnormal CNP regions, they will be considered as a part of abnormal regions and combined with them. Otherwise, they are considered as a normal region and removed. In fact, T2 is an approximation of the CNP region radius and T3 is an approximation of the CNP region diameter.

\subsection{Dataset}

A set of 36 images consisting of 18 pathologic and 18 normal images were used. Pathologic and normal images were acquired from 12 diabetic participants with DR at different stages and 18 healthy volunteers, respectively.

In the patients group, the mean age was $52.82 \pm 11.31$ years and female/male ratio was $4 / 8$. In the normal group, the mean age was $39.07 \pm 8.27$ and female/male ratio was $9 / 9$. The signal strength index ranged from 46 to 69 and 62 to 83 in patient and normal group, respectively.

Images were acquired using a commercial spectral-domain OCT imaging device (RTVue-XR, Optovue, USA). This instrument uses a light source centered on $840 \mathrm{~nm}$ with a full-width half-maximum bandwidth of $45 \mathrm{~nm}$ and has an A-scan rate of $70 \mathrm{kHz}$. The tissue axial resolution of this device is $5 \mu \mathrm{m}$, and the transverse is $15 \mu \mathrm{m}$.

Imaging was performed over $3 \times 3 \mathrm{~mm}$ field-of-view centered on the fovea with a 1.6-mm depth to obtain OCTA volumes consisting of $304 \times 304 \times 512$ voxels. Each data cube was acquired in $\sim 2.9 \mathrm{~s}$. Two repeated B-scans, each containing 304 A-scans, were captured at each fixed position before proceeding to the next sampling location. A three dimensional data cube was formed by orthogonal registration and merging two consecutive scan volumes including one $x$-fast scan and one $y$-fast scan. ${ }^{42}$

Angiography information was extracted using the splitspectrum amplitude-decorrelation angiography algorithm by computing the signal amplitude-decorrelation between two consecutive B-scans of the same location. ${ }^{43}$ 
Table 1 Illustration of the parameter estimation in the dataset.

\begin{tabular}{|c|c|c|c|c|c|c|c|c|}
\hline & \multirow[b]{3}{*}{ VD $\geq 1$} & \multirow[b]{3}{*}{$V D \geq 2$} & \multicolumn{6}{|c|}{$T_{2}=3$} \\
\hline & & & \multirow[b]{2}{*}{$V D \geq 3$} & \multicolumn{5}{|c|}{$R=4$} \\
\hline & & & & $V D \geq 4$ & $V D \geq 5$ & $V D \geq 6$ & $V D \geq 7$ & $V D \geq 10$ \\
\hline MPFN & 30030.22 & 8490.389 & 342.2778 & 21.11111 & 1.333333 & 0 & 0 & 0 \\
\hline MPFD & 42039.39 & 19223.89 & 5660.167 & 2396.834 & 1117.556 & 443.0001 & 231.8334 & 40.78 \\
\hline$\frac{\left(M^{\prime} F D_{V D}-M P F N_{V D}\right)}{M P F D_{V D}}$ & 0.2857 & 0.5583 & 0.9395 & 0.9912 & 0.9988 & 1 & 1 & 1 \\
\hline
\end{tabular}

Using the built-in software, the OCTA volume is segmented to different structural boundaries. Then, by computing maximum flow projection within the slabs defined by the segmented boundaries, en-face projection angiograms, including superficial [between $3 \mu \mathrm{m}$ beneath the internal limiting membrane and $15 \mu \mathrm{m}$ beneath the inner plexiform layer (IPL)], deep (between 15 and $70 \mu \mathrm{m}$ beneath the IPL), and choriocapillaris (between 31 and $59 \mu \mathrm{m}$ beneath the retinal pigment epithelium), are constructed. ${ }^{43,44}$ Since DR influences superficial and deep capillary plexuses, we segmented CNP regions in these two angiograms.

In Optovue OCTA devices, en-face images can be directly extracted in color and gray-level modalities. In this work, we used the red channel of the color modality because it has higher contrast compared to the corresponding grayscale image.

\section{Evaluation}

In this section, first, the evaluation process is explained, which consists of ground truth description, evaluation metrics, and the methods used for comparison. Then, the performance of the proposed method versus the compared methods will be investigated.

\subsection{Evaluation Process}

We evaluated our proposed method both qualitatively and quantitatively. The segmentation results were compared with manual delineation ground truth provided by two trained ophthalmologists (observer 1 and observer 2). Using painting tool of the Photoshop software, the experts manually drew the boundary of each abnormal CNP regions on the color en-face angiogram. Then, using a code we wrote in MATLAB, each color en-face image with manual delineated boundary was converted to the binary image (ground truth). The experts delineated only the boundary of abnormal nonperfusion regions, not all nonperfusion areas.

CNP regions were manually segmented twice by each observer. The variability in the manual segmentations delineated by different observers (interobserver agreement) and by the same observer (intraobserver agreement) were evaluated using Dice similarity coefficient (DSC), which is computed as follows: ${ }^{45}$

$\mathrm{DSC}=2 \times \frac{\left|\mathrm{GT}_{1} \cap \mathrm{GT}_{2}\right|}{\left|\mathrm{GT}_{1}\right|+\left|\mathrm{GT}_{2}\right|}$,

where $\mathrm{GT}_{1}$ and $\mathrm{GT}_{2}$ are two ground truths provided by the same or different observers. $\left|\mathrm{GT}_{1} \cap \mathrm{GT}_{2}\right|$ shows the number of pixels that are nonzero in both ground truths. $\left|\mathrm{GT}_{i}\right|$ shows the number of nonzero pixels in the $i$ 'th ground truth.

In intraobserver variability evaluation, each observer segmented the CNP regions manually in two different sessions. For both observers, the time interval of segmentation between these two sessions was more than 1 month in the superficial plexuses and more than 3 weeks in the deep angiograms.

Table 2 shows the mean \pm standard deviation of DSC for the evaluation of intra- and interobserver agreement in the manual segmentation of CNP regions.

In this table, $\mathrm{Obs} 1_{i}$ and $\mathrm{Obs} 2_{i}$ show the manual ground truth provided by the first and second observer in the $i$ th session,

Table 2 Mean \pm standard deviation of DSC for intra- and interobserver variability evaluation in the manual segmentation of abnormal CNP regions for three groups, including normal, diabetic, and all (normal + diabetic) subjects in the superficial and deep plexuses.

\begin{tabular}{|c|c|c|c|c|}
\hline & \multirow[b]{2}{*}{ Method } & \multicolumn{2}{|c|}{ Intraobserver variability } & \multirow{2}{*}{$\frac{\text { Interobserver variability }}{\mathrm{Obs}_{1 \& 2}-\mathrm{Obs}_{1 \& 2}}$} \\
\hline & & $\mathrm{Obs}_{1}-\mathrm{Obs}_{2}$ & $\mathrm{Obs}_{1}-\mathrm{Obs}_{2}$ & \\
\hline \multirow[t]{3}{*}{ Superficial } & Normal & $0.9532 \pm 0.0173$ & $0.9623 \pm 0.0090$ & $0.9547 \pm 0.0153$ \\
\hline & Diabetic & $0.9376 \pm 0.0341$ & $0.9295 \pm 0.0236$ & $0.8983 \pm 0.0532$ \\
\hline & Normal + Diabetic & $0.9454 \pm 0.0278$ & $0.9459 \pm 0.0242$ & $0.9265 \pm 0.0480$ \\
\hline \multirow[t]{3}{*}{ Deep } & Normal & $0.9687 \pm 0.0182$ & $0.9663 \pm 0.0122$ & $0.9644 \pm 0.0242$ \\
\hline & Diabetic & $0.9445 \pm 0.0374$ & $0.9136 \pm 0.0685$ & $0.8205 \pm 0.0986$ \\
\hline & Normal + Diabetic & $0.9566 \pm 0.0315$ & $0.9399 \pm 0.0554$ & $0.8925 \pm 0.1016$ \\
\hline
\end{tabular}


respectively. Obs $1_{1 \& 2}$ and Obs $2_{1 \& 2}$ are the unions of the both ground truths provided by each of the first and second observers at different sessions, respectively. As can be seen, there is a good intra- and interobserver agreement in our study (a DSC value larger than 0.7 indicates good agreement ${ }^{46}$ ).

We used precision and recall measures to evaluate our proposed method quantitatively. These measures are defined as follows:

Precision $=\frac{\mathrm{TP}}{\mathrm{TP}+\mathrm{FP}}$,

Recall $=\frac{\mathrm{TP}}{\mathrm{TP}+\mathrm{FN}}$,

where TP is the number of abnormal pixels, which were correctly detected; FP is the number of pixels, which were wrongly detected as abnormal regions; and FN is the number of abnormal regions pixels, which were not detected.

The correlation of segmentation was evaluated using Jaccard index $(J)$. Considering the segmented result (SR) obtained by the algorithm and ground truth given with the dataset, $J$ is defined as follows:

$J=\frac{|\mathrm{SR} \cap \mathrm{GT}|}{|\mathrm{SR} \cup \mathrm{GT}|}$,

where $|\mathrm{SR} \cap \mathrm{GT}|$ shows the number of pixels that are nonzero in both SR and GT. $|\mathrm{SR} \cup \mathrm{GT}|$ shows the number of nonzero pixels in the union of SR and GT.

The result of the proposed method was compared with the proposed method by Zhang et al., ${ }^{9}$ which has recently been published for automatic CNP region segmentation and the baseline approach used in the Schottenhamml et al. ${ }^{10}$ method. The comparison was done on our dataset because there is no public available dataset in this field.

In the implementation of Zhang et al. ${ }^{9}$ method, we used the preprocessing steps of the proposed method instead of a reflectance-adjusted thresholding step presented in Ref. 9, because we have not accessed the B-scans in the commercial version of the Optovue device. Therefore, in the preprocessing step, first, vesselness filter was applied to the input image and then the binary vessel mask was obtained using Otsu thresholding. The main and postprocessing steps were implemented based on their published paper. In the main processing, first, a VD map was acquired by applying Euclidean distance transform to the binary vessel mask and then the CNP map was obtained by applying the threshold value $D T=4$ to the VD map. In the postprocessing step, the extracted CNP map was modified using morphological operations. So that, first, the CNP regions were eroded by a five-pixel wide square structuring element and then regions smaller than eight pixel or regions with minor axis length smaller than two pixels were eliminated. Finally, remained regions were dilated by a seven-pixel wide square structuring element. Because of this alternation in the preprocessing step, in the following, this method is called modified Zhang et al. ${ }^{9}$

Another simple and valuable approach, which was used in the Schottenhamml et al., ${ }^{10}$ is the baseline approach. As it was described in Sec. 1, in the baseline approach, first the vessel network is segmented, and then each nonvessel pixel in the logical vessel map is considered as a CNP region.

To have a valid comparison with the baseline approach and denoting the usefulness of watershed algorithm, we used the vessel segmentation map provided by our proposed method using Otsu thresholding rather than the vessel segmentation method used in the Schottenhamml et al. ${ }^{10}$ method. We also used the final circle-checking step to specify abnormal CNP regions for the baseline method. Therefore, the baseline method was implemented in four steps: (1) appling vesselness filter, (2) providing the vessel binary mask using Otsu thresholding, (3) finding the connected components of the inverted binary mask, and (4) obtaining final CNP map by removing any CNP candidates that it was not possible to draw a circle of radius $R=4$ pixels inside them (circle checking).

\subsection{Evaluation of Recall and Precision Rates for the Proposed Method and the Compared Methods}

Table 3 shows the results of precision and recall on all images for our proposed method, the modified Zhang et al. ${ }^{9}$ and the baseline methods by observer $1\left(\mathrm{Obs} 1_{1 \& 2}\right)$, observer 2 $\left(\mathrm{Obs} 2_{1 \& 2}\right)$, and the intersection of observer 1 and observer 2 $\left(O b s 1_{1 \& 2} \cap \mathrm{Obs} 2_{1 \& 2}\right)$ in each of the superficial and deep capillary plexuses. As can be seen, in the superficial (deep) capillary plexus, the proposed method has better performance compared to other methods with recall rate $0.6864(0.7068)$ for Obs $1_{1 \& 2}$, 0.7108 (0.7196) for $\mathrm{Obs} 2_{1 \& 2}$, and 0.7435 (0.8045) for

Table 3 The result of precision and recall on all images for modified Zhang et al., ${ }^{9}$ baseline, and proposed method by Obs $1_{1 \& 2}$, Obs2 ${ }_{1 \& 2}$, and the intersection of them in each of the superficial and deep plexuses.

\begin{tabular}{|c|c|c|c|c|c|c|c|}
\hline & \multirow[b]{2}{*}{ Method } & \multicolumn{2}{|c|}{$O b s 1_{1 \& 2}$} & \multicolumn{2}{|c|}{ Obs2 $_{1 \& 2}$} & \multicolumn{2}{|c|}{$\mathrm{Obs}_{1 \& 2} \cap \mathrm{Obs}_{1 \& 2}$} \\
\hline & & Recall & Precision & Recall & Precision & Recall & Precision \\
\hline \multirow[t]{3}{*}{ Superficial } & Modified Zhang et al. ${ }^{9}$ & 0.2748 & 0.9498 & 0.2856 & 0.9475 & 0.3062 & 0.9445 \\
\hline & Baseline method & 0.9217 & 0.4225 & 0.9259 & 0.4075 & 0.9360 & 0.3830 \\
\hline & Our proposed method & 0.6864 & 0.8522 & 0.7108 & 0.8471 & 0.7435 & 0.8240 \\
\hline \multirow[t]{3}{*}{ Deep } & Modified Zhang et al. ${ }^{9}$ & 0.3953 & 0.9111 & 0.3878 & 0.9079 & 0.4695 & 0.8721 \\
\hline & Baseline method & 0.9156 & 0.4121 & 0.9320 & 0.4261 & 0.9568 & 0.3470 \\
\hline & Our proposed method & 0.7086 & 0.7854 & 0.7196 & 0.8102 & 0.8045 & 0.7186 \\
\hline
\end{tabular}


Table 4 The result of precision and recall rates in normal subjects for modified Zhang et al., ${ }^{9}$ baseline, and proposed method by Obs $1_{1 \& 2}$, Obs $2_{1 \& 2}$, and the intersection of them in each of the superficial and deep plexuses.

\begin{tabular}{|c|c|c|c|c|c|c|c|}
\hline & \multirow[b]{2}{*}{ Method } & \multicolumn{2}{|c|}{ Observer1 } & \multicolumn{2}{|c|}{ Observer2 } & \multicolumn{2}{|c|}{ Observer1 $\cap$ Obsever2 } \\
\hline & & Recall & Precision & Recall & Precision & Recall & Precision \\
\hline \multirow[t]{3}{*}{ Superficial } & Modified Zhang et al. ${ }^{9}$ & 0.5133 & 0.9818 & 0.5525 & 0.9815 & 0.5565 & 0.9815 \\
\hline & Baseline method & 0.9211 & 0.5147 & 0.9592 & 0.4977 & 0.9643 & 0.4968 \\
\hline & Our proposed method & 0.7994 & 0.9955 & 0.8549 & 0.9886 & 0.8610 & 0.9885 \\
\hline \multirow[t]{3}{*}{ Deep } & Modified Zhang et al. ${ }^{9}$ & 0.5456 & 0.9901 & 0.5443 & 0.9890 & 0.5625 & 0.9876 \\
\hline & Baseline method & 0.9381 & 0.7897 & 0.9338 & 0.7871 & 0.9540 & 0.7771 \\
\hline & Our proposed method & 0.8115 & 0.9937 & 0.8087 & 0.9916 & 0.8344 & 0.9886 \\
\hline
\end{tabular}

Table 5 The result of precision and recall rates in diabetic subjects for modified Zhang et al., ${ }^{9}$ baseline, and proposed method by Obs $1_{1 \& 2}$, Obs2 ${ }_{1 \& 2}$ and the intersection of them in each of the superficial and deep plexuses.

\begin{tabular}{|c|c|c|c|c|c|c|c|}
\hline & \multirow[b]{2}{*}{ Method } & \multicolumn{2}{|c|}{ Observer1 } & \multicolumn{2}{|c|}{ Observer2 } & \multicolumn{2}{|c|}{ Observer1 $\cap$ Obsever2 } \\
\hline & & Recall & Precision & Recall & Precision & Recall & Precision \\
\hline \multirow[t]{3}{*}{ Superficial } & Modified Zhang et al. ${ }^{9}$ & 0.2143 & 0.9313 & 0.2207 & 0.9280 & 0.2400 & 0.9232 \\
\hline & Baseline method & 0.9218 & 0.4042 & 0.9178 & 0.3895 & 0.9285 & 0.3604 \\
\hline & Our proposed method & 0.6577 & 0.8160 & 0.6757 & 0.8113 & 0.7125 & 0.7825 \\
\hline \multirow[t]{3}{*}{ Deep } & Modified Zhang et al. ${ }^{9}$ & 0.3527 & 0.8802 & 0.3442 & 0.8763 & 0.4360 & 0.8271 \\
\hline & Baseline method & 0.9092 & 0.3615 & 0.9315 & 0.3777 & 0.9579 & 0.2895 \\
\hline & Our proposed method & 0.6795 & 0.7334 & 0.6948 & 0.7649 & 0.7937 & 0.6511 \\
\hline
\end{tabular}

Obs $1_{1 \& 2} \cap$ Obs $2_{1 \& 2}$ while preserving the high precision rate $0.8522(0.7854)$ for $O b s 1_{1 \& 2}, 0.8471(0.8102)$ for $O b s 2_{1 \& 2}$, and $0.8240(0.7186)$ for $\mathrm{Obs}_{1 \& 2} \cap \mathrm{Obs} 2_{1 \& 2}$.

Considering Obs $1_{1 \& 2} \cap \operatorname{Obs} 2_{1 \& 2}$ as a ground truth, in the superficial (deep) plexus, the proposed method outperformed the compared methods by improving recall rate around $44 \%$ (34\%) compared to the modified Zhang et al. ${ }^{9}$ and precision rate around $44 \%$ (37\%) compared to the baseline method.

Tables 4 and 5 show the precision and recall rates, respectively, in normal and diabetic subjects for the proposed method and the compared methods in each of the superficial and deep plexuses, individually. As can be seen, in the normal subjects, the proposed method outperforms both the modified Zhang et al. ${ }^{9}$ and the baseline methods by decreasing the false positive rate (high precision rate) while preserving recall rate on a high value. In the superficial (deep) plexus of the normal subjects, precision rate was improved around 49\% (21\%) compared to the baseline method and recall rate was improved around $30 \%$ (27\%) compared to the modified Zhang et al. ${ }^{9}$ In the superficial (deep) plexus of the diabetic subjects, compared to the baseline method, precision rate was improved around $42 \%$ (36\%) and compared to the modified Zhang et al. method ${ }^{9}$ recall rate was improved around $47 \%(36 \%)$.

Although it can be observed in Table 5 for diabetic subjects that the precision of the proposed method by the modified
Zhang et al. ${ }^{9}$ is higher (around $14 \%$ in the superficial and $18 \%$ in the deep plexus) compared to our proposed method, the significant difference around $47 \%$ in recall rate shows the drawback of this method in correctly segmenting the regions up to the boundary. And also, it can be observed in the baseline method that the recall rate is higher for both normal (around $10 \%$ in the superficial and $12 \%$ in the deep plexus) and diabetic (around 22\% in the superficial and 16\% in the deep plexus) subjects, although there is a significant difference in precision rate for both normal and diabetic groups compared to the proposed method.

Figures 8 and 9 show the segmentation results of the proposed method and the compared methods using the ground truth of Obs $1_{1 \& 2} \cap \mathrm{Obs} 2_{1 \& 2}$ on two diabetic (Fig. 8) and two normal samples (Fig. 9) of the superficial and deep plexuses. In these figures, the green and dark blue regions show the automatic segmentation results and the ground truth, respectively. The light blue regions show the overlapping of the automatic segmentation and the ground truth.

Figures 8(i)-8(1) show the result of applying the baseline method on two diabetic samples in the superficial and deep plexuses. As can be seen, this method has the highest false positive compared to the other methods due to its simple assumption that considered each nonvessel pixels as an abnormality.

The thresholding-based method proposed by Zhang et al. ${ }^{9}$ failed to extract most of the abnormal regions and correctly 


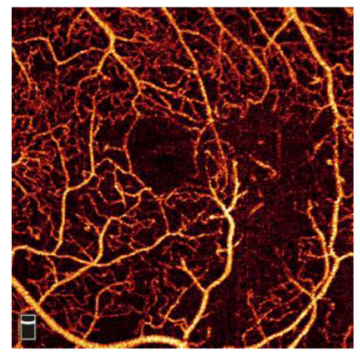

(a)

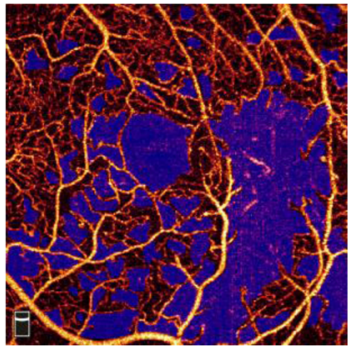

(e)

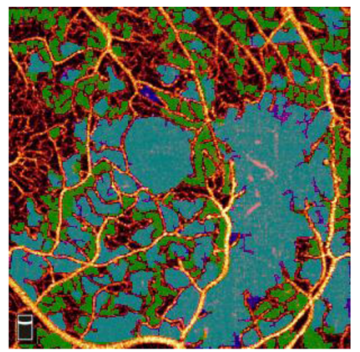

(i)

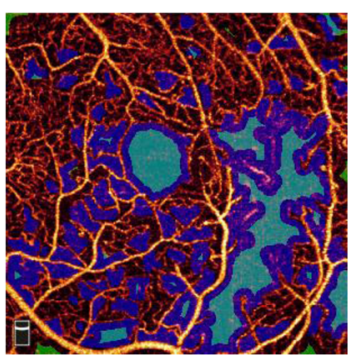

(m)

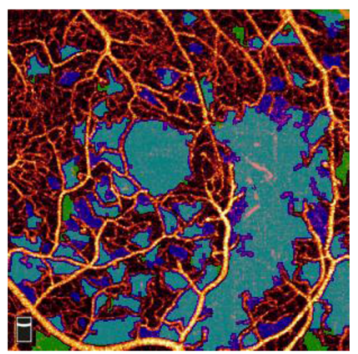

(q)

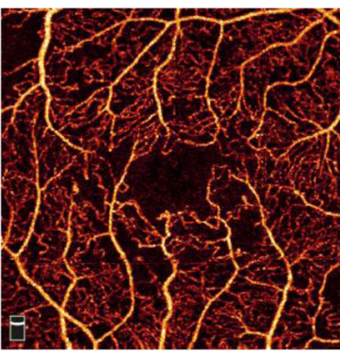

(b)

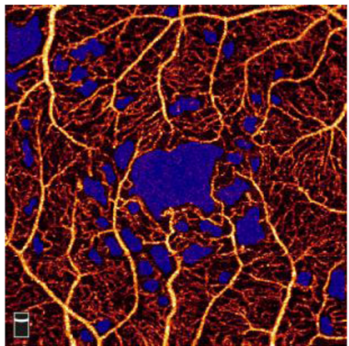

(f)

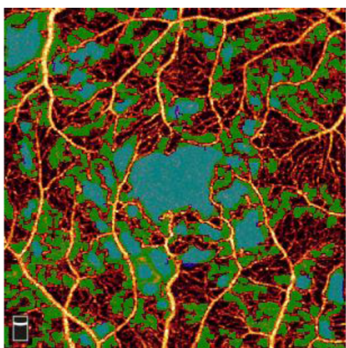

(j)

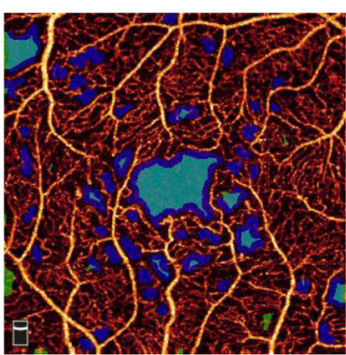

(n)

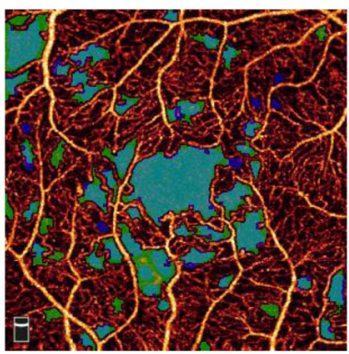

(r)

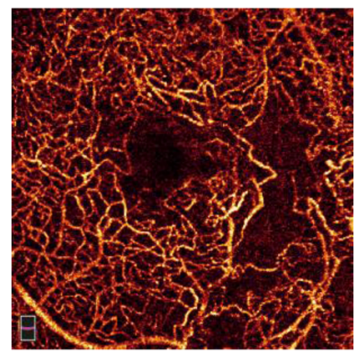

(c)

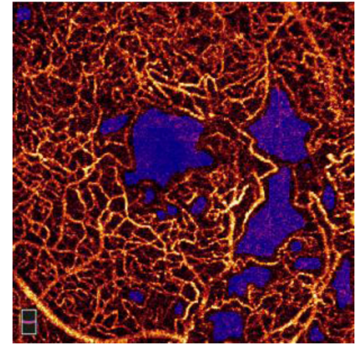

(g)

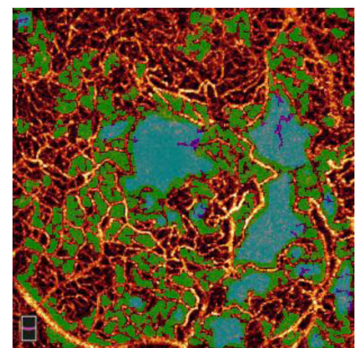

(k)

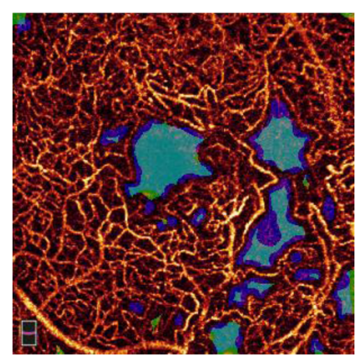

(o)

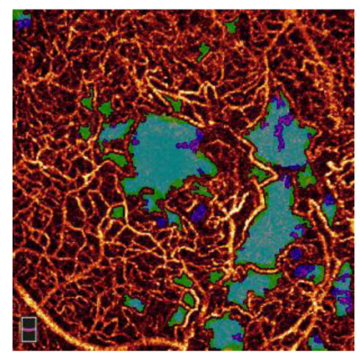

(s)

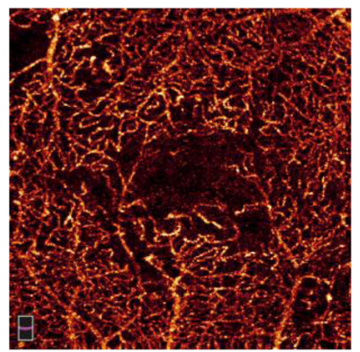

(d)

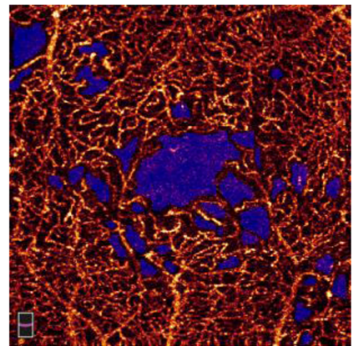

(h)

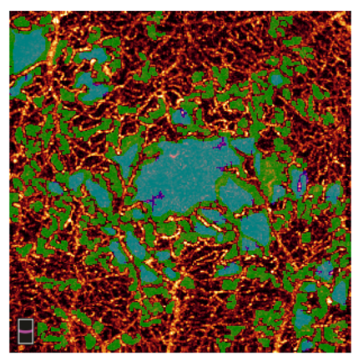

(I)

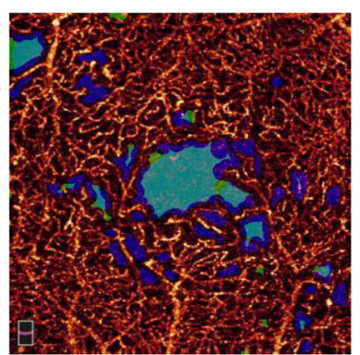

(p)

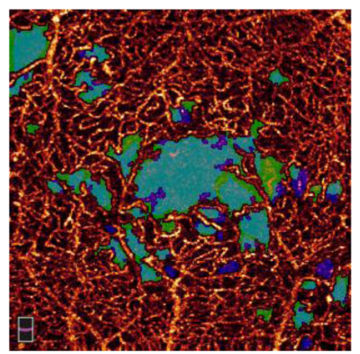

(t)

Fig. 8 A visual comparison between our proposed method and other methods on two samples of diabetic images in each of the $(a, b)$ superficial and $(c, d)$ deep plexus. (a-d) Original diabetic images, $(e-h)$ ground truths, (i-l) baseline method, (m-p) modified Zhang et al., ${ }^{9}$ and (q-t) proposed method.

segment them up to their boundary. However, it had the least false positive regions compared to the other methods [Figs. 8(m)-8(p)].

The proposed method could segment the abnormal CNP regions up to their true boundary more accurately than the compared methods, as shown in Figs. 8(q)-8(t). However, our method segmented some false positive regions due to the extraction of physiologic CNP regions along the large vessels, low-quality OCTA images, and oversegmentation of some abnormal CNP regions. 


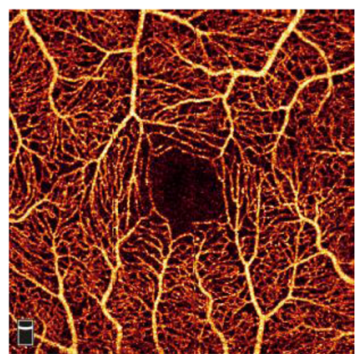

(a)

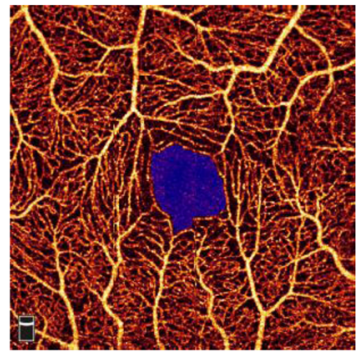

(e)

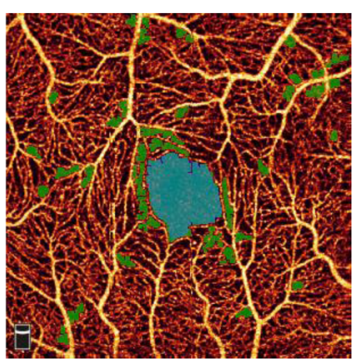

(i)

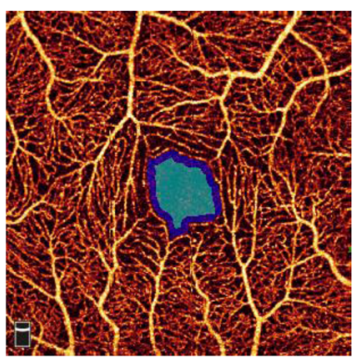

(m)

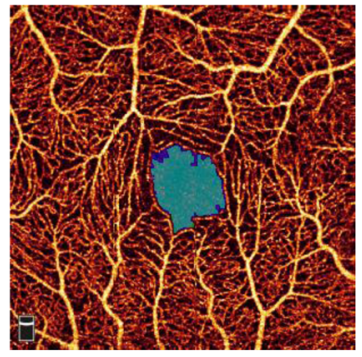

(q)

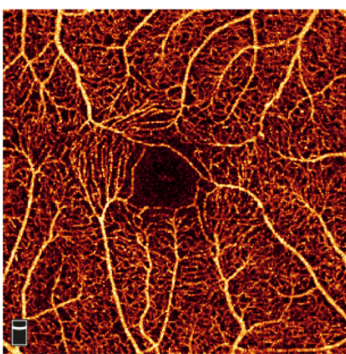

(b)

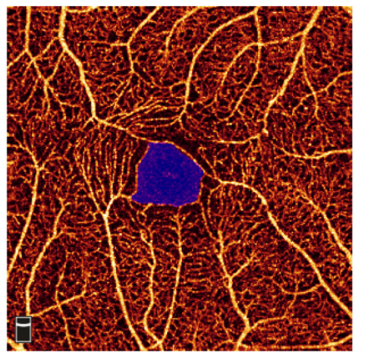

(f)

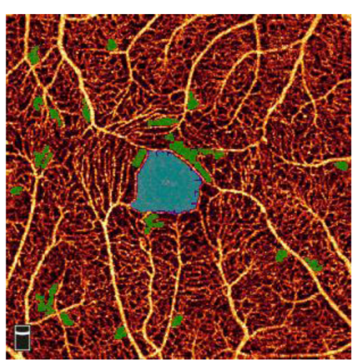

(j)

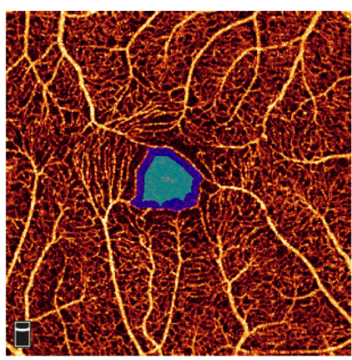

(n)

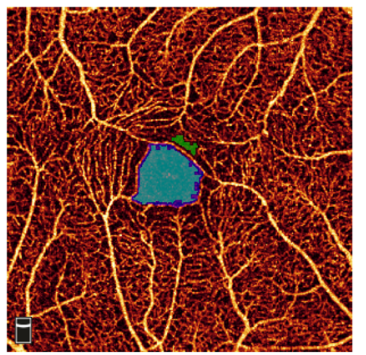

(r)

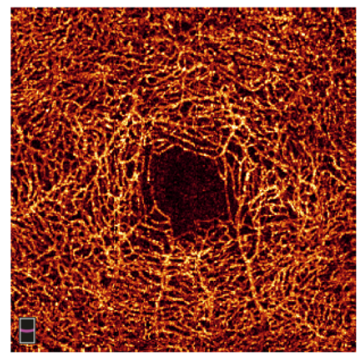

(c)

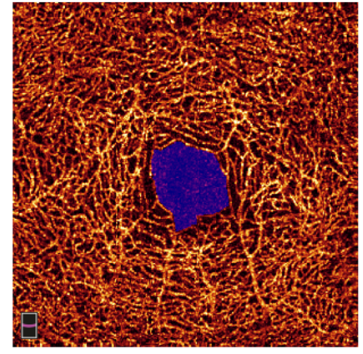

(g)

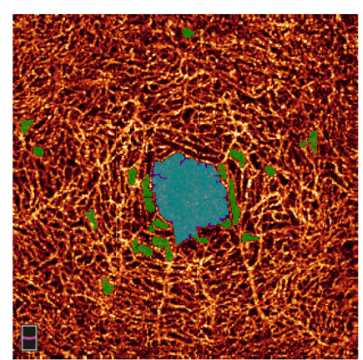

(k)

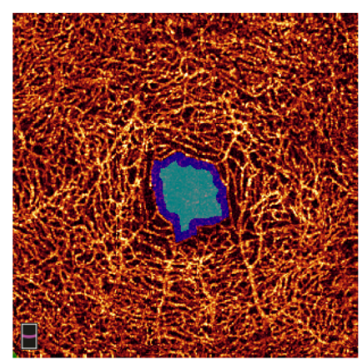

(o)

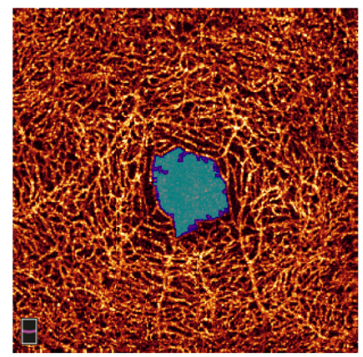

(s)

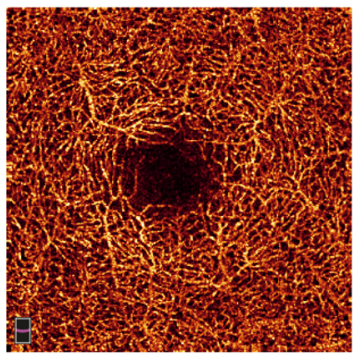

(d)

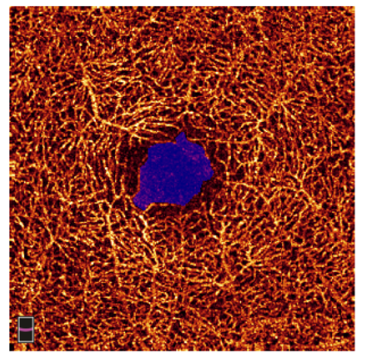

(h)

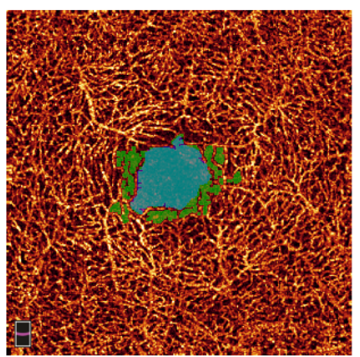

(I)

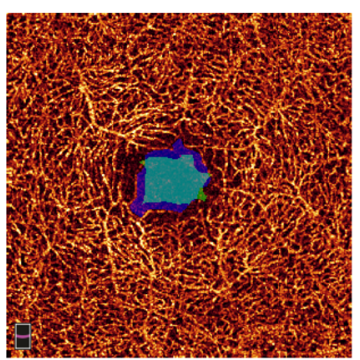

(p)

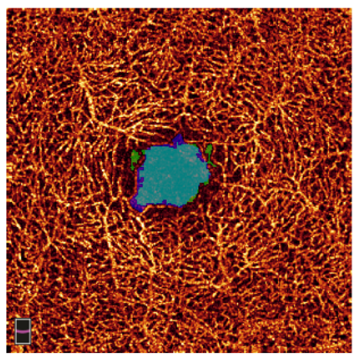

(t)

Fig. 9 A visual comparison between our proposed method and other methods on two samples of normal images in each of the (a,b) superficial and (c,d) deep plexus. (a-d) Original diabetic images, (e-h) ground truths, (i-l) baseline method, $(m-p)$ modified Zhang et al., ${ }^{9}$ and $(q-t)$ proposed method.

Although the FAZ is an anatomical part of the retina, in pathological cases, destruction and enlargement of the FAZ happen. Therefore, we did not remove the FAZ area from the segmented abnormalities.
In the normal subjects (Fig. 9), the FAZ is the only avascular region that should be segmented. As it is clear in Figs. 9(i)-9(1), the baseline method segmented some physiologic regions in addition to the FAZ due to its segmentation strategy, which 
Table 6 Mean \pm standard deviation of $J$ for modified Zhang et al., ${ }^{9}$ baseline, and proposed method by Obs $1_{1 \& 2} \cap$ Obs $2_{1 \& 2}$ in the superficial and deep plexus of three groups, including normal, diabetic, and all (normal + diabetic) subjects.

\begin{tabular}{lllll} 
& \multicolumn{1}{c}{ Method } & \multicolumn{3}{c}{ Jaccard similarity metric } \\
\cline { 3 - 5 } Superficial & Modified Zhang et al. ${ }^{9}$ & Normal & Diabetic & All subjects \\
\cline { 3 - 5 } & Baseline method & $0.5397 \pm 0.0771$ & $0.2399 \pm 0.0815$ & $0.3898 \pm 0.1709$ \\
& Our proposed method & $0.5190 \pm 0.1712$ & $0.3314 \pm 0.0982$ & $0.4252 \pm 0.1672$ \\
Deep & Modified Zhang et al. ${ }^{9}$ & $0.8503 \pm 0.0421$ & $0.5930 \pm 0.0556$ & $0.7217 \pm 0.1392$ \\
& Baseline method & $0.5526 \pm 0.0571$ & $0.3938 \pm 0.0989$ & $0.4732 \pm 0.1132$ \\
& Our proposed method & $0.7533 \pm 0.0947$ & $0.2874 \pm 0.0552$ & $0.5203 \pm 0.2483$ \\
& & $0.8228 \pm 0.0421$ & $0.5690 \pm 0.0693$ & $0.6959 \pm 0.1405$ \\
\hline
\end{tabular}

considered each nonvessel pixel as an abnormality. The method proposed by Zhang et al. ${ }^{9}$ failed to segment the FAZ up to its true boundary due to the use of thresholding segmentation technique [Figs. 9(m)-9(p)].

According to Figs. 9(q)-9(t), our proposed method could segment the FAZ more accurately than the compared methods, although it has one false positive region in Fig. 9(1) because of the low-quality of the input image.

\subsection{Evaluation of the Segmentation Correlation for the Proposed Method and the Compared Methods}

To evaluate segmentation correlation, Jaccard index between the result of the proposed method and ground truths in each of the superficial and deep capillary plexuses was computed.

Table 6 shows the result of Jaccard similarity metric in each of the superficial and deep plexuses of normal, diabetic, and all subjects, for the modified Zhang et al., ${ }^{9}$ the baseline and the proposed method by Obs $1_{1 \& 2} \cap \mathrm{Obs} 2_{1 \& 2}$ as a ground truth. As can be seen, in comparison with the other methods, the proposed method has higher Jaccard rate on all images with a mean of 0.72 and maximum correlation up to 0.86 in the superficial plexus and a mean of 0.70 with a maximum rate of 0.84 in the deep plexus.

\subsection{Evaluation of the Proposed Method and the Compared Methods in Automatic Quantification of Abnormal CNPS}

As it was mentioned previously, automatic quantification of abnormal CNP regions can be helpful in monitoring patient's treatment process. In Refs. 9 and 10, the total avascular area (TAA) and the extrafoveal avascular area (EAA) in normal and pathologic groups were computed to quantify abnormalities. Since the output of the proposed method is a binary map of abnormal CNP regions, TAA was computed by counting the number of nonzero pixels in the binary map. And, same as Zhang et al., ${ }^{9}$ EAA was considered as the avascular area outside the 1-mm central circle.

Table 7 shows the TAA and the EAA values in the superficial and deep plexus of the normal and diabetic subjects for the proposed method, the compared methods, and ground truth $\left(O b s 1_{1 \& 2} \cap \mathrm{Obs} 2_{1 \& 2}\right)$. As can be seen, in both capillary plexuses, for all compared methods, the TAA and EAA are

Table 7 Mean \pm standard deviation of TAA and EAA for modified Zhang et al.., ${ }^{9}$ baseline, ground truth (Obs $1_{1 \& 2} \cap$ Obs2 $\left.2_{1 \& 2}\right)$, and proposed method in the superficial and deep plexuses of the normal and diabetic subjects.

\begin{tabular}{|c|c|c|c|c|c|}
\hline & \multirow[b]{2}{*}{ Method } & \multicolumn{2}{|c|}{ TAA $\left(\mathrm{mm}^{2}\right)$} & \multicolumn{2}{|c|}{ EAA $\left(\mathrm{mm}^{2}\right)$} \\
\hline & & Normal & Diabetic & Normal & Diabetic \\
\hline \multirow[t]{4}{*}{ Superficial } & Ground truth & $0.2927 \pm 0.0829$ & $1.1081 \pm 0.7120$ & 0 & $0.7330 \pm 0.6543$ \\
\hline & Modified Zhang et al. ${ }^{9}$ & $0.1660 \pm 0.0573$ & $0.2881 \pm 0.1875$ & $0.0030 \pm 0.0052$ & $0.1086 \pm 0.1546$ \\
\hline & Baseline method & $0.5683 \pm 0.1810$ & $2.8548 \pm 0.8015$ & $0.2195 \pm 0.1610$ & $2.3484 \pm 0.7688$ \\
\hline & Our proposed method & $0.2550 \pm 0.0737$ & $1.0090 \pm 0.5122$ & $0.0008 \pm 0.0024$ & $0.6701 \pm 0.4654$ \\
\hline \multirow[t]{4}{*}{ Deep } & Ground truth & $0.3134 \pm 0.0814$ & $0.8688 \pm 0.4176$ & 0 & $0.4338 \pm 0.3302$ \\
\hline & Modified Zhang et al. ${ }^{9}$ & $0.1785 \pm 0.0554$ & $0.4580 \pm 0.2743$ & $0.0002 \pm 0.0004$ & $0.1590 \pm 0.1550$ \\
\hline & Baseline method & $0.3848 \pm 0.0978$ & $2.8751 \pm 1.1635$ & $0.0331 \pm 0.0291$ & $2.2671 \pm 1.0828$ \\
\hline & Our proposed method & $0.2645 \pm 0.0727$ & $1.0591 \pm 0.6414$ & 0 & $0.5896 \pm 0.5102$ \\
\hline
\end{tabular}


significantly larger in the diabetic group compared to the normal group.

It can be observed from Table 7 that the proposed method has the closest values of TAA and EAA to the ground truth in each of the superficial and deep plexuses compared to the other methods. In addition, the EAA values in normal images show that the least physiologic regions (false positives) were extracted by the proposed method.

The baseline method has the maximum of TAA and EAA in each normal and diabetic group, which indicates a high false positive rate in this method. And the modified Zhang et al. ${ }^{9}$ method has the least TAA and EAA values due to the high false negative rate in this method.

The proposed method has been implemented in MATLAB 2016a and tested on a Laptop with Intel Core i7-4510U at $2.00 \mathrm{GHz}$ and $8 \mathrm{~GB}$ RAM. The average processing time of the algorithm for each image was $\sim 3.31$ and $4.12 \mathrm{~s}$ in the superficial and deep plexuses, respectively.

\section{Discussion}

In this section, the efficiency of the proposed method in two fields, automatic segmentation of CNP regions and automatic quantification of abnormalities, has been discussed.

\subsection{Efficiency of the Proposed Method in Automatic Segmentation of Abnormal CNP Regions}

In comparison with some state-of-the-art methods, the method proposed here is more flexible and extensible. The proposed method is flexible and extensible because it is possible to extend it based on the new findings in the medical studies of OCTA images by changing the similarity and removing measures in the merging and removing algorithms. The study of CNP regions and their changes in the en-face angiograms of OCTA images is an open medical research field. For example, it is observed in the superficial and deep plexus of diabetic patients that many of the microaneurysms and vessel irregularities are happened in the vicinity of the abnormal CNP regions. ${ }^{19,47}$ Therefore, our result can be improved by extending the proposed method based on these new features.

Another advantage of the proposed postprocessing step is its ability to remove some artifacts created by the vesselness filter in the preprocessing step. By using this filter, the speckle structure is enhanced alongside improving the contrast of the input image [Figs. 4(b) and 4(c)].

As it was shown in Figs. 7(a) and 7(b), these artifacts did not affect the final result because by using merging and removing algorithms in the postprocessing step, they are merged with the nonperfusion regions or they are removed.

As it was shown in the evaluation results, the proposed method has more performance compared to the modified Zhang et al., ${ }^{9}$ and the baseline approach used in the Schottenhamml et al. ${ }^{10}$ In the proposed method by Zhang et al., ${ }^{9}$ the conception of the abnormal CNP regions is almost considered by computing VD map, but finally, abnormal regions are extracted by thresholding this map. In this method, in order to decrease false positive in normal group, the considered threshold value is relatively large that causes some abnormal CNP regions not to be segmented or segmented incompletely. In the baseline approach, after vessel network extraction, all that remains is considered as abnormal CNP regions. This approach not only cannot calculate a trustable amount of the area as a quantification factor of the abnormal CNP regions but also cannot show an accurate map of their position. The reason is that wherever there is the smallest path between CNP regions through vessel network discontinuity, this approach connects the regions and represents them as a large CNP region; this path might occur due to the vessel segmentation error or it was a normal discontinuity in vessel network. Therefore, the efficiency of this approach is highly dependent on the accuracy of the vessel segmentation process while providing an accurate vessel segmentation algorithm in 2-D projected OCTA images is difficult due to the low quality of OCTA images and motion artifacts.

OCTA imaging devices are progressing toward improving signal-to-noise ratio and enhancing image quality but the software that is included in the commercial versions of this device is not properly capable of eliminating noise and managing the distortions caused by the patient movement. Although this motion artifact can be reduced by using the tracking module, imaging with this mode takes longer time than patient tolerance. Therefore, proposing a method that is less sensitive to low-quality images is valuable. The core of the proposed method (the watershed algorithm) is sensitive to noise, but we could significantly reduce this sensitivity by computing foreground and background marker images in the preprocessing step and using merging and removing procedures in the postprocessing step.

Overall, our proposed automatic abnormal CNP segmentation method has the following advantages:

(1) Being fast and completely automatic.

(2) Efficient mapping between the definition of abnormal CNP regions and the concept of catchment basin in the watershed algorithm.

(3) Extracting the regions with more accurate boundary.

(4) The possibility of taking advantage of expert knowledge by considering postprocessing step.

\subsection{Efficiency of the Proposed Method in Automatic Quantification of Abnormal CNP Regions}

Different parameters are used for quantifying capillary perfusion alternation in the macular disease that can be categorized into two groups. The first group is vessel density-based parameters used in Refs. 11, 48, and 49. The second group is nonperfusion area-based parameters used in Refs. 9, 10, 47, and 49. A study has been recently published, which shows that the nonperfusion area-based parameters may be more sensitive to microvascular impairment compared to the vessel density-based parameters. ${ }^{49}$

All nonperfusion area-based parameters for clinical studies can be computed using the abnormal CNP map provided by our proposed method. In addition, in comparison with other nonperfusion area-based methods, our proposed method has the following advantages:

(1) Unlike Ref. 49, only the abnormal CNP regions are extracted and the normal distance between vessels are not considered.

(2) The proposed method is more robust to small errors occurring in the vessel segmentation compared to the proposed methods in Ref. 10. Because the proposed method is based on the conception of abnormal CNP regions and uses the merging and removing algorithms to obtain final abnormal CNP regions. 


\section{Conclusion and Future Work}

In this paper, we proposed an automatic abnormal CNP region segmentation method based on the marker-controlled watershed algorithm. Our proposed method has three main steps. In the first step, the input image was enhanced using vesselness filter and then the foreground and background marker images were computed. In the second step, the marker images were imposed in the gradient magnitude of the preprocessed image and then the CNP region candidates were obtained by applying the watershed algorithm to the imposed image. And finally, in the third step, correct abnormal CNP regions were obtained by modeling candidates as an undirected weighted graph and using merging and removing procedures.

The proposed method was evaluated on the superficial and deep plexuses of OCTA images dataset consisting of 18 normal and 18 diabetic subjects acquired from spectral-domain OCTA imaging. The results showed that our proposed method outperformed the state-of-the-art method with the recall rate of 0.74 and precision rate of 0.82 in the superficial plexus and the recall rate of 0.80 and precision rate of 0.72 in the deep plexus. Although the proposed method could segment the abnormal CNP regions up to their boundary more accurately, the false positive of the proposed method is considerable. It is expected that using new features like the irregularity of the vessels around regions or the presence or absence of microaneurysms around them in the merging and removing step can reduce the false positive of the proposed method. We investigate it as a future work.

\section{Disclosures}

We have no conflicts of interests.

\section{References}

1. T. S. Hwang et al., "Optical coherence tomography angiography features of diabetic retinopathy," Retina 35(11), 2371-2376 (2015).

2. D. A. Antonetti et al., "Diabetic retinopathy: seeing beyond glucoseinduced microvascular disease," Diabetes 55(9), 2401-2411 (2006).

3. G. Quellec et al., "Deep image mining for diabetic retinopathy screening," Med. Image Anal. 39, 178-193 (2017).

4. Group ETDRSR, "Early treatment diabetic retinopathy study design and baseline patient characteristics: ETDRS report number 7," Ophthalmology 98(5), 741-756 (1991).

5. C. Agurto et al., "Multiscale AM-FM methods for diabetic retinopathy lesion detection," IEEE Trans. Med. Imaging 29(2), 502-512 (2010).

6. R. Ganjee, R. Azmi, and M. Ebrahimi Moghadam, "A novel microaneurysms detection method based on local applying of markov random field," J. Med. Syst. 40(3), 1-9 (2016).

7. Group ETDRSR, "Classification of diabetic retinopathy from fluorescein angiograms: ETDRS report number 11," Ophthalmology 98(5), 807-822 (1991).

8. Group ETDRSR, "Fluorescein angiographic risk factors for progression of diabetic retinopathy: ETDRS report number 13," Ophthalmology 98(5), 834-840 (1991).

9. M. Zhang et al., "Automated quantification of nonperfusion in three retinal plexuses using projection-resolved optical coherence tomography angiography in diabetic retinopathy," Invest. Ophthalmol. Vis. Sci. 57(13), 5101-5106 (2016).

10. J. Schottenhamml et al., "An automatic, intercapillary area-based algorithm for quantifying diabetes-related capillary dropout using optical coherence tomography angiography," Retina 36, S93-S101 (2016).

11. Y. Hwang et al., "Automated quantification of capillary nonperfusion using optical coherence tomography angiography in diabetic retinopathy," JAMA Ophthalmol. 67(3), 223-230 (2014).

12. W. M. Abdelmoula, S. M. Shah, and A. S. Fahmy, "Segmentation of choroidal neovascularization in fundus fluorescein angiograms," IEEE Trans. Biomed. Eng. 60(5), 1439-1445 (2013).
13. R. Ganjee, R. Azmi, and B. Gholizadeh, "An improved retinal vessel segmentation method based on high level features for pathological images," J. Med. Syst. 38(9), 108 (2014).

14. M. R. K. Mookiah et al., "Computer-aided diagnosis of diabetic retinopathy: a review," Comput. Biol. Med. 43(12), 2136-2155 (2013).

15. A. H. Kashani et al., "Optical coherence tomography angiography: a comprehensive review of current methods and clinical applications," Prog. Retinal Eye Res. 60, 66-100 (2017).

16. D. Huang et al., "Optical coherence tomography," Science 254(5035), 1178-1181 (1991).

17. M. Wu et al., "Automatic subretinal fluid segmentation of retinal SDOCT images with neurosensory retinal detachment guided by enface fundus imaging," IEEE Trans. Biomed. Eng. 65(1), 87-95 (2018).

18. L. Yeung et al., "Correlation between spectral domain optical coherence tomography findings and fluorescein angiography patterns in diabetic macular edema," Ophthalmology 116(6), 1158-1167 (2009).

19. K. Sambhav, S. Grover, and K. V. Chalam, "The application of optical coherence tomography angiography in retinal diseases," Surv. Ophthalmol. 62(6), 838-866 (2017).

20. M. Soares et al., "Comparison of diabetic retinopathy classification using fluorescein angiography and optical coherence tomography angiography," Br. J. Ophthalmol. 101(1), 62-68 (2017).

21. A. C. S. Tan et al., "An overview of the clinical applications of optical coherence tomography angiography," Eye 32(2), 262-286 (2017).

22. S. S. Gao et al., "Quantification of choroidal neovascularization vessel length using optical coherence tomography angiography," J. Biomed. Opt. 21(7), 076010 (2016).

23. Z. Chu et al., "Quantitative assessment of the retinal microvasculature using optical coherence tomography angiography," J. Biomed. Opt. 21(6), 066008 (2016).

24. N. Eladawi et al., "Automatic blood vessels segmentation based on different retinal maps from OCTA scans," Comput. Biol. Med. 89, 150-161 (2017).

25. L. Liu et al., "Automated choroidal neovascularization detection algorithm for optical coherence tomography angiography," Biomed. Opt. Express 6(9), 3564-3576 (2015).

26. C. Dongye et al., "Automated detection of dilated capillaries on optical coherence tomography angiography," Biomed. Opt. Express 8(2), 11011109 (2017).

27. M. Zhang et al., "Projection-resolved optical coherence tomographic angiography," Biomed. Opt. Express 7(3), 816-828 (2016).

28. A. F. Frangi et al., "Multiscale vessel enhancement filtering," in Medical Image Computing and Computer-Assisted Interventation (MICCAI'98), Vol. 1496, pp. 130-137 (1998).

29. S. Xu, H. Liu, and E. Song, "Marker-controlled watershed for lesion segmentation in mammograms," J. Digit. Imaging 24(5), 754-763 (2011).

30. C. M. Lo et al., "Multi-dimensional tumor detection in automated whole breast ultrasound using topographic watershed," IEEE Trans. Med. Imaging 33(7), 1503-1511 (2014).

31. W. Gómez et al., "Computerized lesion segmentation of breast ultrasound based on marker-controlled watershed transformation," Med. Phys. 37(1), 82-95 (2010).

32. G. N. Girish, A. R. Kothari, and J. Rajan, "Marker controlled watershed transform for intra-retinal cysts segmentation from optical coherence tomography B-scans," Pattern Recognit. Lett. (2018).

33. J. Yan et al., "Marker-controlled watershed for lymphoma segmentation in sequential CT images," Med. Phys. 33(7), 2452-2460 (2006).

34. Y. Cui et al., "Malignant lesion segmentation in contrast-enhanced breast MR images based on the marker-controlled watershed," Med. Phys. 36(10), 4359-4369 (2009).

35. R. Romero-Zaliz and J. F. Reinoso-Gordo, "An updated review on watershed algorithms," in Studies in Fuzziness and Soft Computing, C. Cruz Corona, Ed., Vol. 358, pp. 235-258, Springer International Publishing, Cham (2018).

36. P. K. Saha, R. Strand, and G. Borgefors, "Digital topology and geometry in medical imaging: a survey," IEEE Trans. Med. Imaging 34(9), 19401964 (2015).

37. J. Roerdink and A. Meijster, "The watershed transform: definitions, algorithms and parallelization strategies," Fundam. Informaticae 41(1-2), 187-228 (2000).

38. N. Otsu, "A threshold selection method from gray-level histograms," IEEE Trans. Syst. Man Cybern. 9(1), 62-66 (1979). 
39. C. R. Maurer, R. Qi, and V. Raghavan, "A linear time algorithm for computing exact Euclidean distance transforms of binary images in arbitrary dimensions," IEEE Trans. Pattern Anal. Mach. Intell. 25(2), 265-270 (2003)

40. P. Soille, Morphological Image Analysis: Principles and Applications, 2nd ed., Springer-Verlag New York, Inc., Secaucus, New Jersey (2003).

41. K. Parvati, B. S. Prakasa Rao, and M. Mariya Das, "Image segmentation using gray-scale morphology and marker-controlled watershed transformation," Discrete Dyn. Nat. Soc. 2008, 1-8 (2008).

42. M. F. Kraus et al., "Motion correction in optical coherence tomography volumes on a per A-scan basis using orthogonal scan patterns," Biomed. Opt. Express 3(6), 1182-1199 (2012).

43. Y. Jia et al., "Split-spectrum amplitude-decorrelation angiography with optical coherence tomography," Opt. Express 20(4), 4710-4725 (2012).

44. R. K. Wang et al., "Three dimensional optical angiography," Opt. Express 15(7), 4083-4097 (2007).

45. L. R. Dice, "Measures of the amount of ecologic association between species," Ecology 26(3), 297-302 (1945).

46. A. P. Zijdenbos et al., "Morphometric analysis of white matter lesions in MR images: method and validation," IEEE Trans. Med. Imaging 13(4), 716-724 (1994).

47. D. A. Salz et al., "Select features of diabetic retinopathy on sweptsource optical coherence tomographic angiography compared with fluorescein angiography and normal eyes," JAMA Ophthalmol. 134(6), 644-650 (2016).

48. S. A. Agemy et al., "Retinal vascular perfusion density mapping using optical coherence tomography angiography in normals and diabetic retinopathy patients," Retina 35(11), 2353-2363 (2015).
49. P. L. Nesper et al., "Quantifying microvascular abnormalities with increasing severity of diabetic retinopathy using optical coherence tomography angiography," Invest. Ophthalmol. Vis. Sci. 58(6), BIO307-BIO315 (2017).

Razieh Ganjee received her MSc degree in computer engineering in the field of artificial intelligence from University of Alzahra, Tehran, Iran. She is a PhD candidate at the Shahid Beheshti University in the field of artificial intelligence, Tehran, Iran. Her research interest includes image processing especially medical image processing consisting of retinal OCT and OCTA images.

Mohsen Ebrahimi Moghaddam is an associate professor in the Computer Science and Engineering Department, Shahhid Beheshti University of Iran, since 2006. He has obtained his PhD, MSc, and BSc degrees from Sharif University in Iran. He is the head of Image Processing Lab in Shahid Beheshti University. His research interests are in image processing and pattern recognition specially using artificial intelligence techniques, such as image security, watermarking, blurring. He is also interested in operating system fields, such as scheduling and security. He has several publications in these fields of research in the journals and conferences.

Ramin Nourinia is an assistant professor of ophthalmology at Shahid Beheshti University, University of Medical Sciences, Tehran, Iran, since 2009 . He is a retina specialist since 2008 . His research interest includes retinal vascular disorders, diabetic retinopathy, retinopathy of prematurity, and retinal imaging, especially OCT and OCTA. He received world ophthalmology congress (WOC) 2018 award. 